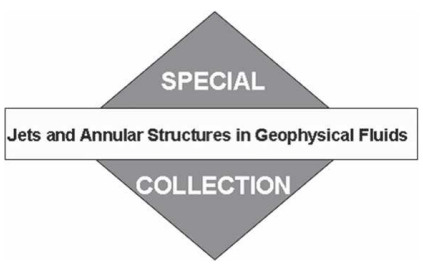

\title{
Forced-Dissipative Shallow-Water Turbulence on the Sphere and the Atmospheric Circulation of the Giant Planets
}

\author{
R. K. SCOTT \\ Northwest Research Associates, Inc., Bellevue, Washington \\ L. M. Polvani \\ Department of Applied Physics and Applied Mathematics, Columbia University, New York, New York
}

(Manuscript received 8 May 2006, in final form 18 January 2007)

\begin{abstract}
Although possibly the simplest model for the atmospheres of the giant planets, the turbulent forceddissipative shallow-water system in spherical geometry has not, to date, been investigated; the present study aims to fill this gap. Unlike the freely decaying shallow-water system described by Cho and Polvani, equilibrium states in the forced-dissipative system are highly dependent on details of the forcing and the dissipation. For instance, it is found that for a given equilibrated energy level, the steadiness of zonal jets depends crucially on the balance between forcing and dissipation.

With long (up to 100000 days) high-resolution (T170) calculations, the dependence of the equilibrium states on Rossby number Ro and Rossby deformation radius $L_{D}$ is explored, for the case when the dissipation takes the form of hypodiffusion (acting predominantly at large scales) and the random forcing at small scales is $\delta$ correlated in time. When $L_{D}$ is large compared to the planetary radius, zonal jets are verified to scale closely with the Rhines scale over a wide range of Ro; furthermore, the jets at the equator are found to be both prograde and retrograde with approximately equal likelihood. As $L_{D}$ is decreased, the equatorial jets become increasingly and consistently retrograde, in agreement with the freely decaying turbulence results. Also, the regime recently discussed by Theiss, where zonal jets are confined to low latitudes, is illustrated to emerge robustly in the limit of small $L_{D}$. Finally, specific calculations with parameter values typical of the giant planets are presented, confirming many of the earlier results obtained in the freely decaying case.
\end{abstract}

\section{Introduction}

The banded appearance of the giant planets and their rich atmospheric features remain largely unexplained. One line of research, pioneered by Busse (1976), has argued that the alternating bands are the surface signature of convective circulations that extend deep into those planets, with concentric cylinders rotating parallel to the planetary axis of rotation and intersecting the planets' surface. While appealing at first sight, in practice this idea has proven difficult to simulate numerically. For one thing, much remains unknown about the planets' interior (Guillot 1999), implying that many assumptions need to be made about the planets' compo-

Corresponding author address: R. K. Scott, Northwest Research Associates, Inc., P.O. Box 3027, Bellevue, WA 98009-3027.

E-mail: scott@nwra.com sition and many largely unconstrained parameters (e.g., the diffusivity in the interior) need to be chosen to perform actual simulations. Furthermore, most published computations with such models (e.g., Heimpel et al. 2005; Stanley and Bloxham 2004; Sun et al. 1993) have, to date, used a Boussinesq approximation, implying the density is nearly constant with radius, surely an unrealistic assumption for the massive giant planets.

Alternatively, a different line of research, pioneered by Williams (1978), has sought to explain the key observed features using a much simpler idea. Assuming that the bands and embedded vortices are the manifestation of a shallow atmospheric circulation, twodimensional or nearly geostrophic equations have been used to model the thin atmospheric layers of the giant planets. Such studies are closely related to the extensive literature on two-dimensional and geostrophic turbulence, and have tried to explain the formation of the 
bands in terms of an arrest of the upward energy cascade owing to strong rotation (Rhines 1975). In addition to requiring considerably more modest computational resources, these models require a much smaller number of free parameters to be chosen for practical computations.

Following this simpler idea, by using the shallowwater equations Cho and Polvani (1996a,b) showed that, in the absence of forcing, an initially random flow on the sphere spontaneously organizes itself into a banded configuration, with the observed number of bands roughly appearing for each of the four giant outer planets once the radius, rotation rate, and Rossby radius are specified. One notable shortcoming of that model, however, concerns the direction of the zonal winds at low latitudes: while Jupiter and Saturn have strong prograde jets at the equator, the freely evolving flows in that model always generate a retrograde equatorial jet. More importantly however, the model of Cho and Polvani suffers from a fundamental limitation: it represents a freely evolving (i.e., unforced) system. Given that the bands have been observed for hundreds of years and are very robust, it is much more likely they result from a forced-dissipative system (rather than a decaying one).

Extending the results of Cho and Polvani to the forced-dissipative case is the main goal of the present study. We start in section 2 by reviewing some key ideas in geostrophic turbulence. While a great deal of work has been carried out with nondivergent $2 \mathrm{D}$ flows (i.e., with an infinite deformation radius) or with divergent nearly 2D flows in doubly period or channel geometries, we submit that, for purposes of understanding the banded appearance of the giant planets, a minimal model needs to have the following three characteristics: it should be in spherical geometry, deal with a fluid with a finite deformation radius, and represent a forceddissipative system. We are aware of no paper in the literature that has presented results for such a system.

This paper then proceeds as follows. In section 3 we describe the physical details of our model (forceddissipative shallow-water equations on the sphere) in a general context. In section 4 we explore how different forcing functions can yield quite different results, an issue that is usually ignored, even in simpler 2D forceddissipative turbulence studies. In section 5 we present the results of our simulations, showing how banded structures appear as the forcing amplitude is decreased, and how finite deformation radius leads to equatorial confinement of the zonal jet, an idea suggested by Theiss (2004) but not previously demonstrated via direct numerical computation in spherical geometry. Further details of our forced-dissipative turbulent flows (e.g., cyclone-anticyclone asymmetry) are discussed in section 6 . In section 7 we refocus our attention on the giant planets, and present forced-dissipative calculations using observed planetary values for the physical parameters. A brief summary and discussion concludes the paper.

\section{Review of geostrophic turbulence}

Geostrophic turbulence is characterized by the simultaneous presence of both turbulent and wavelike motions. Elementary considerations based on the advective nature of potential vorticity (PV) and the principle of invertibility suggest that mixing of PV by the turbulent eddies will eventually lead to distinct latitudinal regions dominated by vortical motion where the $\mathrm{PV}$ is homogenized, separated by sharper jumps of PV on which the motion has a more wavelike character (e.g., McIntyre 1982, and references therein). According to this view, the zonal mean PV field develops naturally into a staircase structure; the flow is anisotropic and typically dominated by narrow zonal jets along the jumps in PV. The length $L_{\mathrm{Rh}} \sim(2 U / \beta)^{1 / 2}$, where $U$ is a typical velocity and $\beta$ is the planetary vorticity gradient, was introduced by Rhines (1975) as the scale at which the flow ceases to be dominated by isotropic motion.

Rhines's original scaling was for $2 \mathrm{D}$ barotropic motion, but the procedure is the same for equivalent barotropic motion, or finite deformation radius. When the effect of the $\beta$ term in the vorticity equation is weak, that is, at scales for which the advection of relative vorticity dominates the advection of planetary vorticity, the time scale, $\tau$, for turbulent motion at wavenumber scale $k$ is given by

$$
\tau \sim(k U)^{-1}
$$

for a typical velocity scale $U$. On the other hand, when the $\beta$ term is significant, the background PV gradient supports Rossby waves with frequency of magnitude:

$$
\omega_{\mathrm{Ro}}=\frac{k \beta}{k^{2}+l^{2}+L_{D}^{-2}} .
$$

Anisotropy will develop at scales for which the turbulent motion projects significantly onto Rossby wave motions, that is, for $\tau^{-1} \sim \omega_{\mathrm{Ro}}$, or at wavenumber

$$
k_{\mathrm{Rh}} \sim \sqrt{\frac{\beta}{2 U}(1-\alpha)},
$$

where $\alpha=U / \beta L_{D}^{2}$. When $L_{D}^{2}<U / \beta(\alpha<1)$, there is no overlap between the Rossby wave and turbulent eddy frequencies and the flow should remain isotropic (Okuno and Masuda 2003; Smith 2004; Theiss 2004). 
In the forced-dissipative case, (3) does not determine $k_{\mathrm{Rh}}$ a priori, since $U$ generally depends in a nontrivial way on both the energy injection and the large-scale dissipation. In the case $L_{D} \rightarrow \infty$, an alternative formulation was proposed by Maltrud and Vallis (1991) that was based instead on the upscale energy flux $\varepsilon$. In an isotropic, energy-cascading inertial range, the typical eddy time scale at wavenumber $k$ can be written in terms of the kinetic energy spectrum $E(k) \sim \varepsilon^{2 / 3} k^{-5 / 3}$ as

$$
\tau \sim\left[k^{3} E(k)\right]^{-1 / 2} .
$$

Equating $\tau^{-1}$ with $\omega_{\text {Ro }}$ gives

$$
k_{\mathrm{Rh}} \sim\left(\frac{\beta^{3}}{\varepsilon}\right)^{1 / 5}
$$

This expression is useful because in practice it is often easier to constrain a priori the upscale energy flux than the total energy, which depends also on the large-scale dissipation. An extension of (5) to finite $L_{D}$ was considered by Smith (2004). Recent studies also have focused on the effect of large-scale dissipation on the scaling of jets at equilibrium (e.g., Danilov and Gurarie 2002, 2004; Smith 2004; Sukoriansky et al. 2007).

The $\beta$-plane approximation has limited application to planetary atmospheres because of order one variations of Coriolis parameter with latitude, $\phi$. On the sphere, both $\beta$ and $L_{D}$ increase as $\phi \rightarrow 0$ and decrease as $\phi \rightarrow$ $\pi / 2$. Specifically, we have $\beta=2 \Omega \cos \phi$ and $L_{D}=$ $(g H)^{1 / 2} / 2 \Omega \sin \phi$. A local application of the planar expression in (3) indicates that, for small enough $L_{D}$, the motion will be anisotropic at low latitudes where $1-$ $\alpha>0$, and isotropic at high latitudes where $1-\alpha<0$ (Theiss 2004). Theiss also demonstrated the existence of this regime using a modified $\beta$-plane model that permitted latitudinal variations of deformation radius in a self-consistent manner. Since $L_{D}$ diverges at the equator, it would appear that there will always be a latitude band near the equator for which (3) is satisfied, even for very large $U / \beta$. However, for consistency near the equator one must substitute the equatorial deformation radius $L_{\mathrm{eq}} \equiv\left(a L_{D}\right)^{1 / 2}$, where $L_{D}=(g H)^{1 / 2} / 2 \Omega$ is the polar deformation radius, $a$ is the planetary radius, and $H$ is the mean layer depth.

Treating $L_{D}$ as an external parameter, the dispersion relation for the modified Rossby-Haurwitz wave is

$$
\omega_{\mathrm{Ro}}=\frac{2 \Omega m}{n(n+1)+a^{2} / L_{D}^{2}},
$$

where $m$ and $n$ are the azimuthal and total wavenumbers, respectively, in the expansion in spherical harmonics. This is an ad hoc expression but it is useful for comparison with the $\beta$-plane results. Equating $\omega_{\mathrm{Ro}}$ with the turbulent frequency, $\tau^{-1} \sim n U / a$ gives

$$
n_{\mathrm{Rh}} \sim \sqrt{\frac{a \Omega}{U}(1-\alpha)},
$$

where now $\alpha=a U / \Omega L_{D}^{2}$, which is valid provided $L_{D}^{2}>a U / \Omega$. In deriving (7) we have used $n \sim 2 m$ for isotropic wavenumbers. Because the Rossby-Haurwitz waves are global modes, this analysis does not include a dependence of the Rhines scale on the local planetary rotation gradient [i.e., only $\Omega$ appears in (7)]. A local analysis may be more appropriate in cases where there are many alternating jets between pole and equator. In the limit $L_{D} \rightarrow \infty$ the spherical equivalent of (5) is, from (6),

$$
n_{\mathrm{Rh}} \sim\left(\frac{\Omega^{3}}{\varepsilon}\right)^{1 / 5} .
$$

In section 5, we verify that (8) holds to a good approximation across a wide range of energy fluxes in the shallow-water system with large $L_{D}$.

Many recent numerical studies of geostrophic turbulence in spherical geometry lend support to the above scaling arguments. In the freely decaying case both barotropic (Yoden and Yamada 1993) and shallowwater (Cho and Polvani 1996a,b; Iacono et al. 1999b) dynamics have been considered. Applied to the four giant planets Cho and Polvani (1996b) found that the scale of zonal jets in shallow-water dynamics was consistent with the observed zonal mean winds. Another study (Cho and Polvani 1996a) found that the divergent nature of shallow-water motion affects the turbulent structure and that cyclone-anticyclone asymmetry is important at small $L_{D}$ (see also Iacono et al. 1999a). The freely decaying case is attractive because it requires no assumptions about forcing or large-scale dissipation and final equilibria depend only on the initial conditions, but it clearly has limitations. All of the above studies found that equatorial motion is dominated by retrograde flow, in contrast to observations of the zonal mean flow on Jupiter and Saturn. Further, because a fraction of the initial energy is invariably lost to small-scale diffusion, and equilibration in general occurs over very long time scales, freely decaying turbulence tends to produce weakly energetic flows and weak, undular jets. More importantly, the freely decaying problem has limited application to planetary atmospheres where energy is continually resupplied, perhaps through thermal convection from the planetary interior.

Studies of the forced-dissipative case, on the other hand, have so far been restricted to barotropic dynam- 
ics. Huang and Robinson (1998) and Nozawa and Yoden (1997) obtained zonal jets that were very steady in time, with the jet spacing decreasing with decreasing forcing amplitude. Other studies showed differences in the power spectra of zonal and background vorticity fields and found some similarities with zonal wind spectra obtained from the giant planets (Galperin et al. 2001; Huang et al. 2001; Sukoriansky et al. 2002). The issue of how dissipation influences jet spacing has also been considered recently by Sukoriansky et al. (2007) who argued that it is frictional processes rather than the Rhines effect that provides the actual halting mechanism of the inverse energy cascade. It has also been shown that both prograde and retrograde equatorial jets occur with roughly equal likelihood (Dunkerton and Scott 2007). However, in view of estimates of $L_{D}$ on the giant planets (e.g., around $L_{D} / a \approx 0.025$ for Jupiter or Saturn; e.g., Cho et al. 2001; Ingersoll et al. 2004), the barotropic model has limited application to those atmospheres.

\section{Physical model and numerical procedure}

We consider the shallow-water system on the sphere, forced at small scales in the vorticity equation by an isotropic random process, with small-scale dissipation in the form of hyperdiffusion (to control the forward enstrophy cascade) and various forms of large-scale dissipation (to allow equilibration of total energy). In terms of the vorticity, $\zeta$, divergence, $\delta$, and height $h$, the equations are

$$
\begin{aligned}
\zeta_{t}+\boldsymbol{\nabla} \cdot\left(\mathbf{u} \zeta_{a}\right) & =F+D_{\zeta}, \\
\delta_{t}-\mathbf{k} \cdot \boldsymbol{\nabla} \times\left(\mathbf{u} \zeta_{a}\right) & =-\nabla^{2}(E+g h)+D_{\delta,} \\
h_{t}+\boldsymbol{\nabla} \cdot(\mathbf{u} h) & =D_{h},
\end{aligned}
$$

where $\zeta_{a}=f+\zeta$ is the absolute vorticity, $f=2 \Omega \sin \phi$ is the Coriolis parameter, $\mathbf{u}$ is the velocity, $E=|\mathbf{u}|^{2} / 2$, and $g$ is gravity. The terms $F$ and $D_{\xi}$, for $\xi \in\{\zeta, \delta, h\}$, represent forcing and dissipation terms, respectively.

The important physical parameters for this system are the Rossby and Froude numbers, defined as

$$
\operatorname{Ro}=\frac{U}{2 a \Omega} \text { and } \operatorname{Fr}=\frac{U}{\sqrt{g H}},
$$

respectively, where $U$ is a typical velocity scale. Either of these can be replaced by the Burger number, $\mathrm{Bu}=$ $\mathrm{Ro}^{2} / \mathrm{Fr}^{2}=\left(L_{D} / a\right)^{2}$, where $L_{D}=(g H)^{1 / 2} / 2 \Omega$ is the polar deformation radius. We nondimensionalize all quantities as follows: horizontal lengths are scaled by the planetary radius $a$ and time is scaled by the planetary rota- tion period $2 \pi / \Omega$, so, in particular, a time of $t=1$ corresponds to one planetary rotation.

The forcing $F$ is a small-scale, random process centered around a fixed total wavenumber $n_{f}=N / 4$, where $N$ is the maximum total wavenumber, designed such that the rate of energy input into the system, $\varepsilon_{0}$, is constant. [As shown in Scott (2007), the inverse cascade is sensitive to the ratio $N / n_{f}$, which should not be too small.] The spectrum of $F$ is given by

$$
\hat{F}(n)=\left\{\begin{array}{cc}
\varepsilon_{0} / \Delta n & \left|n-n_{f}\right| \leq \Delta n / 2 \\
0 & \text { otherwise }
\end{array}\right.
$$

where $\Delta n=4$. The time dependence of the forcing is Markovian, determined by a decorrelation time scale $c_{r}$ with $0 \leq c_{r}<\infty$. There are two limiting cases, corresponding to $\delta$-correlated or white-in-time forcing (for $c_{r}=0$ ) and steady forcing (for $c_{r} \rightarrow \infty$ ). In the former case, a constant kinetic energy input of $\varepsilon_{0}$ is achieved by setting

$$
\hat{f}_{m n}=\sqrt{\frac{2 n(n+1) \varepsilon_{0}}{(2 n+1) \delta t \Delta n}} e^{i \theta},
$$

where $\hat{f}_{m n}$ is the spectral coefficient of $F, m$ is the azimuthal or zonal wavenumber, $\delta t$ is the time step, and $\theta$ is a random phase. For the case of Markovian forcing with $c_{r} \gg \delta t$, a standard formulation is used, starting with

$$
\tilde{f}_{m n}(t+\delta t)=\left(1-r^{2}\right)^{1 / 2} e^{i \theta}+r \tilde{f}_{m n}(t),
$$

where $r$ is related to the decorrelation radius by $c_{r}=$ $\delta t /(1-r)=0.1$. The $\hat{f}_{m n}$ are then defined by

$$
\hat{f}_{m n}=\varepsilon_{0} \tilde{f}_{m n} /\left\langle\tilde{f}_{m n} \tilde{\zeta}_{m n}^{*}\right\rangle,
$$

where $\langle\cdot\rangle$ denotes a global average and $*$ is the complex conjugate. Note that in the shallow-water system these formulations are only approximate because the energy is not a quadratic quantity.

The dissipation, $D_{\xi}$, comprises scale-selective hyperand hypodiffusion, acting at small and large scales, respectively:

$$
D_{\xi}=-\left[\nu_{h}(-\Delta)^{\delta_{h}}+\nu_{l}(-\Delta)^{\delta_{l}}\right] \xi .
$$

The subscripts $h$ and $l$ refer to the dissipation acting on high and low wavenumbers, respectively. For the small scales we use $\delta_{h}=4$ and $\nu_{h}=10 /[N(N+1)]^{\delta_{h}}$ (i.e., a damping time scale of 0.1 on the highest wavenumbers). For the large-scale dissipation, three choices are discussed in section 4: (i) a Rayleigh friction with $\delta_{l}=0$; (ii) a radiative relaxation, again with $\delta_{l}=0$ but applied only to the perturbation height field (i.e., $D_{\zeta} \equiv D_{\delta} \equiv 0$ ), thereby relaxing the perturbation height field to zero 
without any form of mechanical damping; and (iii) a standard hypodiffusion with $\delta_{l}=-1$. The diffusion coefficients in the three cases are $\nu_{l}=0.0001, \nu_{l}=1$, and $\nu_{l}=0.01$, respectively, which ensures an approximate equal equilibrated energy between the cases. In the following section we examine how the total equilibrated energy and hence the typical velocity scale $U$ and Rossby number Ro depends on the energy input $\varepsilon_{0}$ and the dissipation parameters.

The numerical model, whose full details can be found in Rivier et al. (2002) and Scott et al. (2004), uses a pseudospectral horizontal discretization of (9). The time discretization is a standard leapfrog scheme with forcing and dissipation terms treated implicitly, and the time step is adaptively adjusted based on the CourantFriedrichs-Lewy condition. The spectral resolution in most of the calculations presented is T170, that is, the maximum resolved total wavenumber is 170 , corresponding to a $512 \times 256$ longitude $\times$ latitude grid. Some simulations have also been performed at T341, to verify that the results obtained are independent of numerical truncation.

\section{Dependence on forcing and dissipation}

Recent studies of $\beta$-plane turbulence have considered equilibration of forced-dissipative turbulence by large-scale dissipation (e.g., Danilov and Gurarie 2002; Smith 2004). Danilov and Gurarie (2004) also considered the effects of different types of large-scale dissipation operators and found that there is a distortion of the energy inertial range similar to that found in twodimensional turbulence (e.g., Borue 1994; Sukorianski et al. 1999), when dissipation is restricted to the large scales. For the special case in which the large-scale dissipation takes the form of a linear drag, Danilov and Gurarie (2002) and Smith (2004) provided estimates for the jet scale in terms of the drag coefficient and energy input. In spherical geometry, Sukoriansky et al. (2007) provide a detailed analysis of jet scaling in the case of the forced barotropic system with linear drag. So far there has been no systematic analysis of the effects of forcing and dissipation in shallow-water turbulence in spherical geometry.

In this section, we explore the extent to which the forcing and large-scale dissipation affect the equilibrium states. We seek physically meaningful choices of forcing and dissipation that lead to steady, equilibrated jets, to allow a comparison in section 5 of the characteristics of equilibria for different physical parameters. In the present discussion we focus on the case of large deformation radius, $L_{D}=10$, to minimize divergent effects, and consider a representative value of energy

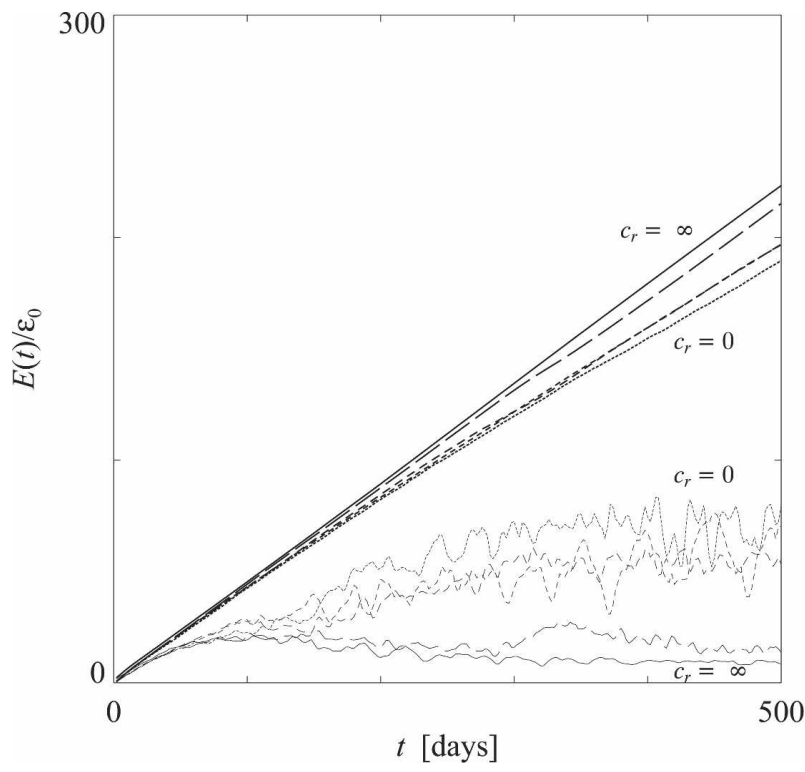

FIG. 1. Total kinetic energy normalized by input rate vs time for the decorrelation radius $c_{r}=0,1,10,100, \infty$ (increasing dash lengths from dotted to solid). Bold lines denote total energy, light lines denote energy in the azonal wavenumber sector, $m>n / 5$.

input, $\varepsilon_{0}=0.1 \times 10^{-6}$. The many different forms and combinations of forcing and dissipation mechanisms make this a large problem; here we do not attempt a comprehensive treatment but provide some basic examples of how the turbulent flow depends on these choices.

\section{a. Energy injection}

Our forcing is designed to represent the actual forcing of the giant planetary atmospheres by overturning convective systems or other random motions generated by the deep turbulent interior. There is very little known about these processes and so a simple set of assumptions is required. Keeping these to a minimum, we suppose that the forcing is random in space and time; uniform, homogeneous, and isotropic in space; and induces motions primarily at scales smaller than those of the dominant features of the atmospheres. For convenience we also require that the forcing can inject energy into the system at an easily controlled rate. The reader is referred to Showman (2007) for further discussion and an example of physical space forcing.

Forcing at small scales gives rise to an inverse energy cascade through an inertial range where the effects of forcing and dissipation are small. We choose the forcing to be Markovian in time and arranged such that the total energy input is constant. Figure 1 shows the energy evolution for different values of the decorrelation time scale, $c_{r}$, from 0 for delta-correlated (white in 
time) forcing to $\infty$ for steady forcing. The energy growth is approximately linear up to $t=500$ (i.e., 500 planetary rotations). The actual total energy transferred upscale, $\varepsilon$, in other words, the energy input $\varepsilon_{0}$ minus the energy lost to the small-scale hyperdiffusion, can be calculated from the slopes of the lines, giving $\varepsilon$ between about $0.4 \varepsilon_{0}$ and $0.45 \varepsilon_{0}$. Increasing the distance in spectral space between the input scale and truncation wavenumber results in a greater fraction of $\varepsilon_{0}$ being transferred upscale, but reduces the separation between the large scales and the input scale.

The light lines in Fig. 1 show the "azonal" component of the energy, arising from spectral modes for which $m>n / 5$. [Note that this definition differs from that of Huang et al. (2001, their Fig. 1), where the azonal sector comprised "segment" wavenumbers. The current definition includes all isotropic wavenumbers, only excluding wavenumbers near the zonal axis, and is a more natural representation of the eddy field.] The azonal kinetic energy is a much larger fraction of the total energy for the cases with $c_{r} \leq 10$ : over a third of the energy is contained in the azonal modes, compared with less than a tenth for $c_{r} \geq 100$. In other words, a larger fraction of the energy input into the system remains in the eddy modes, and hence at small scales, before organizing into zonal modes. In turn, more energy at small scales implies stronger dissipation by the small-scale hyperdiffusion, which accounts for the systematic reduction in $\varepsilon$ with decreasing $c_{r}$. Examination of the spatial dependence of the vorticity field (not shown) for the steady and $\delta$-correlated cases confirms that there is a more active eddy field in the latter case.

Since steady forcing is difficult to motivate physically, we conducted our main calculations using the values $c_{r}=10$ and $c_{r}=0$. In fact, despite the small differences at early times, the longtime behavior is very similar between these two choices, and so we report only the case $c_{r}=0$.

Of course, there are many other factors that determine the form of the energy injection: the forcing could be applied to the divergence or height fields rather than the vorticity field, as here; it could also be applied in physical space rather than in spectral space; it could be designed as a representation of outflow from convective systems, or of the stirring induced by a horizontal shear instability; the scale of the forcing and the spectral or physical space distribution must also be chosen. A detailed study of these factors is beyond the scope of the present work.

\section{b. Large-scale dissipation}

One of the commonest mechanisms for removing energy in forced turbulence is a linear drag, or Rayleigh (a)

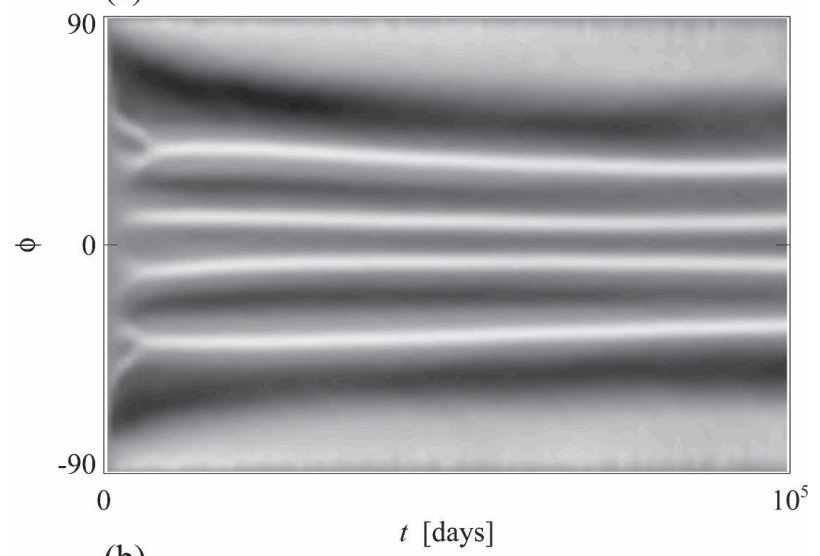

(b)

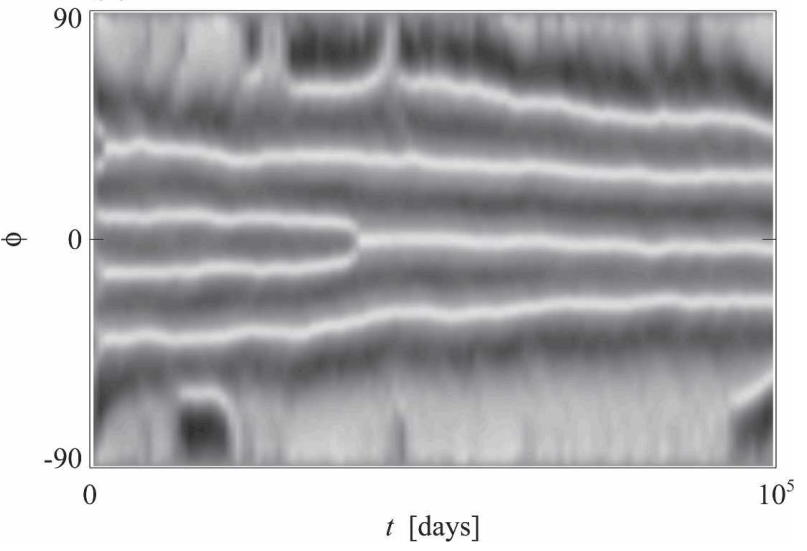

FIG. 2. Zonal velocity vs latitude and time for large-scale dissipation modeled by Rayleigh friction, with energy input and friction coefficient $\left(\varepsilon_{0}, \nu_{l}\right)=\mu\left(0.1 \times 10^{-6}, 10^{-4}\right)$, with multiplier (a) $\mu=1$ and (b) $\mu=10$; deformation radius $L_{D}=10$. The total energy in each case is approximately the same. Gray scale is normalized according to the rms value, with light (dark) shade indicating eastward (westward) flow.

friction. Figure 2 a shows the time evolution of the zonal mean zonal velocity for a typical case. Zonal jets (light denotes eastward) quickly form and reach a quasi equilibrium, with very small and gradual drift of the jets over time. The total energy reaches equilibrium by about $t=10^{4}$ days.

Undoubtedly, the biggest motivation for using Rayleigh friction in turbulence studies is that it provides a simple closure for the total energy of the system. For a given net energy input $\varepsilon$ and a Rayleigh friction coefficient $\nu_{l}$ (in the notation of section 3 ), the total equilibrated energy in the barotropic system is given by $\mathcal{E}=$ $\varepsilon / 2 \nu_{l}$. This closure allows a certain amount of analytic progress in the barotropic case and enables the Rhines scale to be predicted from (7) a priori for a given forcing and dissipation strength (e.g., Danilov and Gurarie 2002; Smith 2004). 
However, the equilibrated total energy by no means fully characterizes the equilibrium flow. Different flows with the same $\mathcal{E}$ can be readily obtained by multiplying both the energy input and the friction coefficient by the same factor. Figure $2 b$ shows a case identical to the previous one, but with energy input and friction multiplied by a factor of 10 . Consistent with expectations from scaling, the number of jets in the domain in each case is the same, being determined by total energy of the flow through (7), which differs only by around $10 \%$ between the two cases. However, although the number of jets is the same, the character of the jets is completely different, remaining very steady in time in the first case, but exhibiting larger short-term variability manifested in meandering jets in the second. Note how the direction of the equatorial flow changes from retrograde to prograde at around $t=4 \times 10^{4}$.

Figure 3 shows the instantaneous vorticity field at $t=$ $10^{5}$ for the two cases illustrated in Fig. 2. There is much greater small-scale eddy activity in the second case than in the first, where more of the energy organizes into zonal bands. In the second case, the balance between energy input and removal is attained at smaller scales. We emphasize that equilibrium does not depend uniquely on the equilibrated total energy, but that, on the contrary, very different flow fields can be obtained with similar total energy depending only on the strength of forcing and dissipation.

In the context of a rotating fluid in contact with a rigid surface or ground Rayleigh friction has natural justification in terms of the Ekman pumping due to the frictional boundary layer. On the other hand, for an atmospheric layer not in contact with a frictional ground Rayleigh friction, which damps momentum equally at all scales, is less justified (e.g., Smith 2004). As a representation of dissipation in the atmospheres of the giant planets, Rayleigh friction may be of marginal relevance, notwithstanding the possibility of magnetohydrodynamic damping in the deeper atmosphere. In the context of planetary atmospheres a more physically motivated large-scale dissipation arises from radiative relaxation, which we consider next.

An example of a case using radiative relaxation as large-scale dissipation is shown in Fig. 4a. This case is otherwise similar to the Rayleigh friction case shown in Fig. 4a. An interesting feature of the evolution is that the flow at low latitudes increases continually throughout the integration. The reason for this is that radiative relaxation places only a very weak constraint on the angular momentum in low latitudes (e.g., Garcia 1987; Haynes 1998; Scott and Haynes 1998). In the present case, forcing continually injects energy into the system, (a)

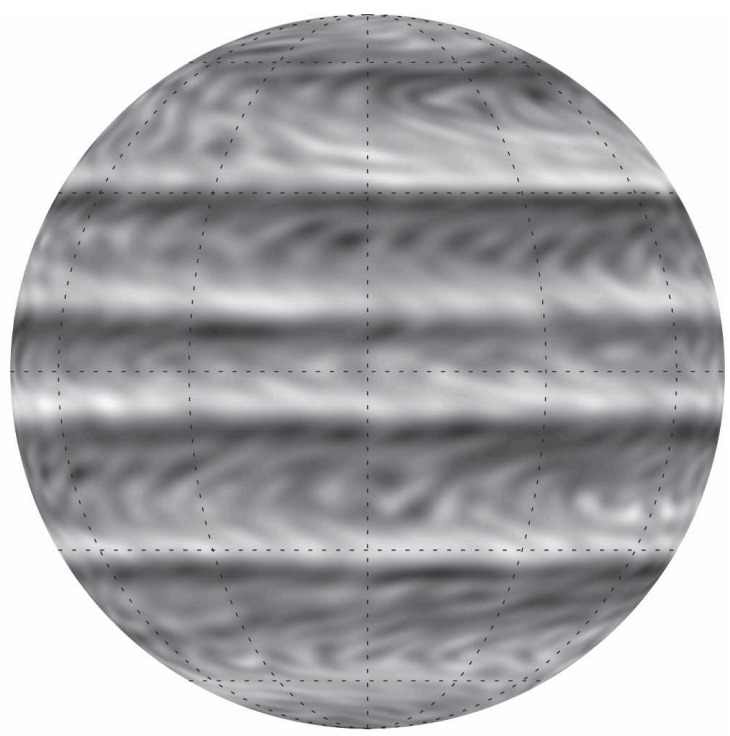

(b)

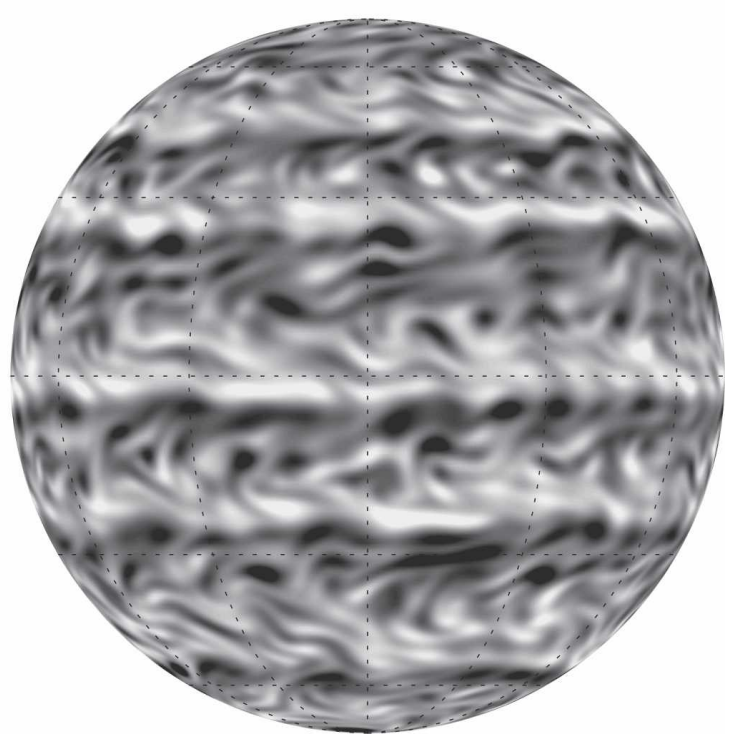

FIG. 3. Vorticity field at $t=10^{5}$ days for the cases shown in Fig. 2. Orthographic projection; light shades correspond to positive vorticity.

which then accumulates in the zonal flow. The flow in midlatitudes is damped by the effect of the relaxation on the streamfunction, but in the Tropics the relation between height and streamfunction is weaker and there is less damping of the flow there. Note that the equatorial jet is both stronger than the midlatitude jets and prograde, two features in common with the zonal mean velocity profiles of the giant planets.

Radiative relaxation is commonly used to model atmospheric flows but appears not to have been considered previously in more idealized studies of two- 
(a)

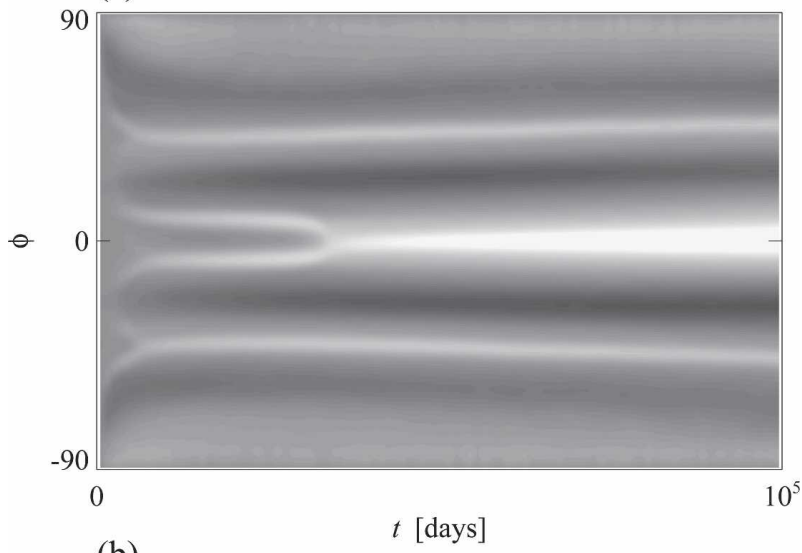

(b)

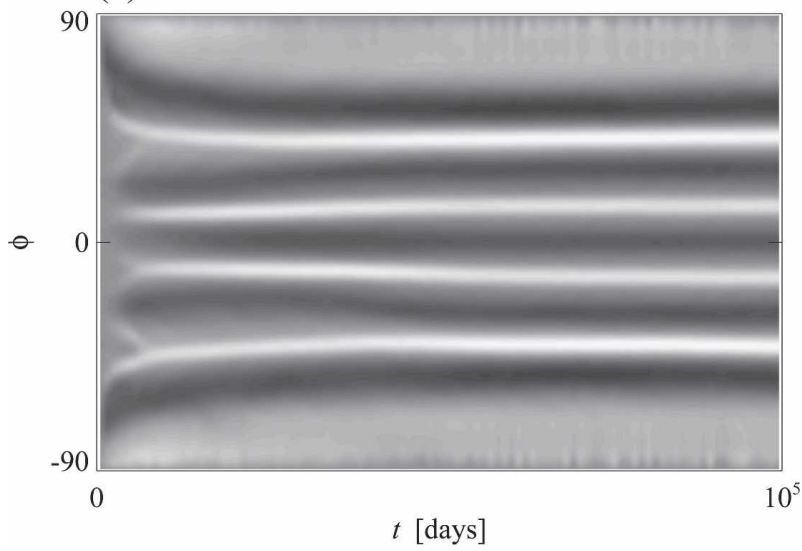

FIG. 4. Zonal velocity vs latitude and time for different forms of large-scale dissipation: (a) radiative relaxation with relaxation coefficient $\nu_{l}=1$ and (b) hypodiffusion with diffusion coefficient $\nu_{l}=0.01$. Energy input is the same as in Fig. 2a. Gray scale is normalized according to the rms value, with light (dark) shade indicating eastward (westward) flow.

dimensional equivalent barotropic or shallow-water turbulence. One possible reason is that, unlike Rayleigh friction, there is no obvious relation between the radiative relaxation coefficient and the equilibrated total energy. Indeed, even the process of equilibration is unclear in spherical geometry, because of the weak constraint on angular momentum at low latitudes. In shallow water, radiative relaxation is represented by a relaxation to zero of the perturbation height field, that is, a term $-v_{l} h$ on the right hand side of $(9 \mathrm{c})$. In midlatitudes, as on the $\beta$ plane, this is equivalent to a linear relaxation on the streamfunction. Further, because the streamfunction is related to the vorticity through the inverse Laplacian, a relaxation on the streamfunction is intrinsically more confined to larger scales than the linear vorticity drag of Rayleigh friction described above. Radiative relaxation therefore provides a means to remove energy at large scales without affecting smaller scales, and in a way that has natural physical motivation; see also Showman (2007) for a discussion of the radiative balance on Jupiter. The drawback in spherical geometry appears to be an exceedingly slow rate of equilibration at low latitudes.

The third form of large-scale dissipation is a simple hypodiffusion, an example of which is shown in Fig. 4b. We have chosen the diffusion coefficient to be such as to give approximately the same equilibrated total energy as in the case with the Rayleigh friction, although now there is no simple closure that can determine the energy a priori on the basis of the forcing and diffusion. Compared with Fig. 2a we see that the number of jets in the domain is similar, consistent with idea that this is determined by the total energy. In other aspects, such as the structure of the jets, the two calculations are also similar.

At first sight, hypodiffusion has no physical motivation, though it has the useful property of restricting dissipation effects to the largest scales only. In fact, for the special case used here where the hypodiffusion takes the form of an inverse Laplacian operator $\Delta^{-1}$ [i.e., $\delta_{l}=-1$ in (15)], there is a natural correspondence with radiative relaxation, insofar as $\Delta^{-1} q=\psi$ (in the case of 2D barotropic flow), and the hypodiffusion is then also equivalent to a relaxation on the streamfunction. Thus, as far as midlatitude dynamics is concerned, hypodiffusion with $\delta_{l}=-1$ is a good approximation to radiative relaxation. In the Tropics, hypodiffusion damps vorticity at the same rate as in midlatitudes and therefore avoids the build up of low-latitude momentum obtained with radiative relaxation.

We conclude this section with a consideration of how the equilibration of the total energy itself varies across all three forms of dissipation. In Fig. 5 we show the time evolution of total energy (normalized by energy input rate) for a range of energy input rates $\varepsilon_{0}=(100,10,1$, $0.1,0.01) \times 10^{-6}$, for the restricted time range $0 \leq t \leq$ $10^{4}$; the inset shows the extended time range $0 \leq t \leq 10^{5}$ corresponding to the cases shown in Fig. 2a and Fig. 4 $\left(\varepsilon_{0}=0.1 \times 10^{-6}\right)$. In all cases, the largest contribution to the energy is from the zonal wavenumbers (cf. bold and light lines) and only a fraction of the total energy is contained in eddy motion.

For the case of Rayleigh friction, the relation between total energy and energy input hold to a good approximation across the full range of input values (four orders of magnitude). In the other cases, there is no such relation and the curves do not collapse. For the case of radiative relaxation, the lack of equilibration is clear in all cases, although the total (normalized) energy growth is slower when the energy input rate is larger. As the inset shows, there is still approximately 
(a)

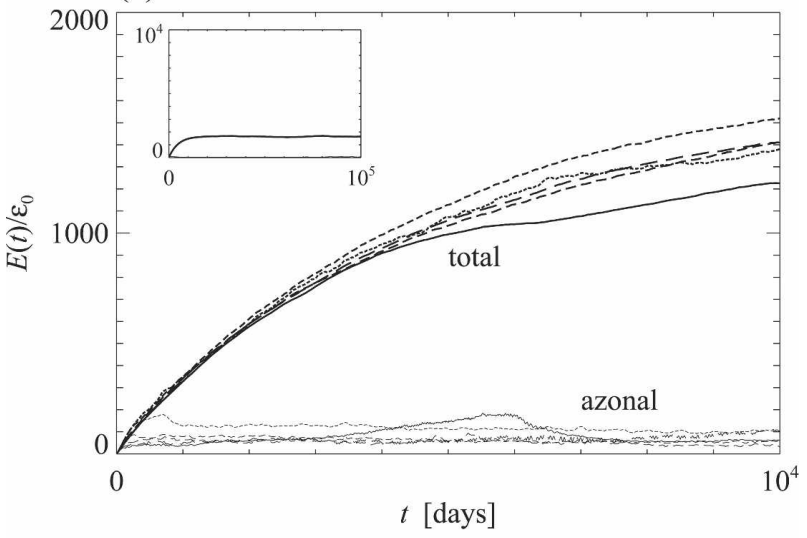

(b)

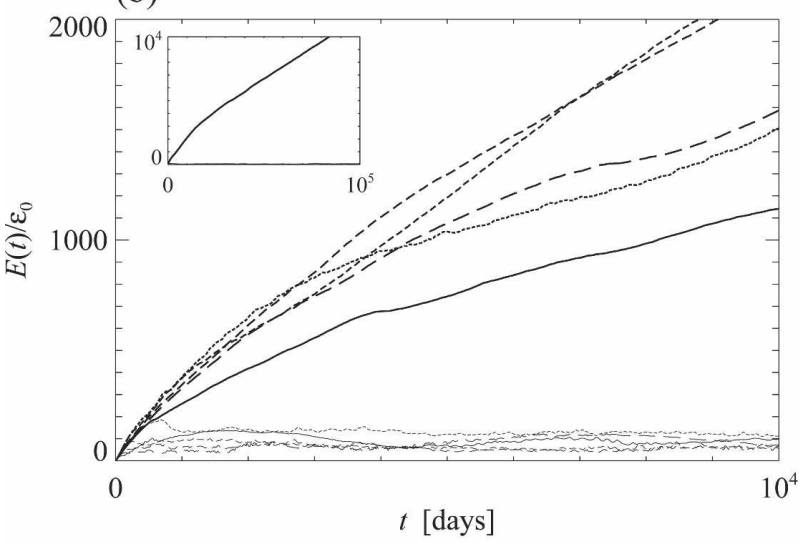

(c)

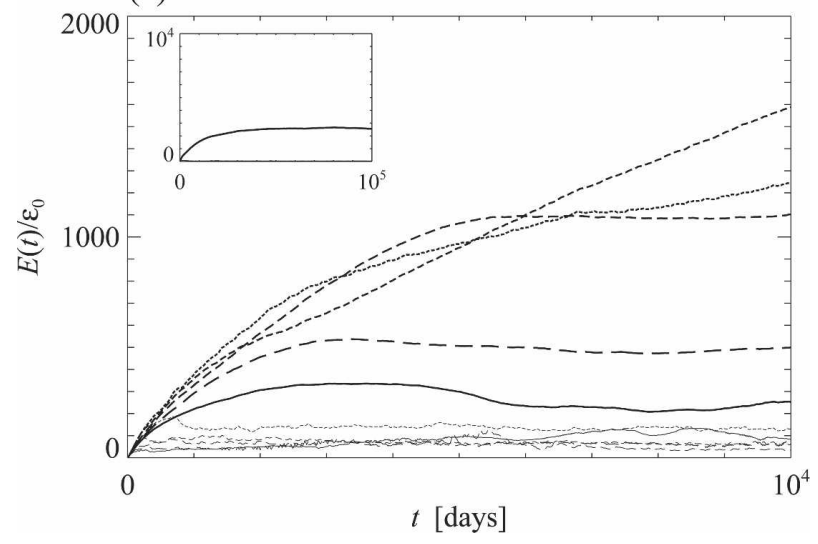

FIG. 5. Total kinetic energy normalized by input rate vs time for $\varepsilon_{0}=(0.01,0.1,1,10,100) \times 10^{-6}$ (increasing dash lengths from dotted to solid) for each dissipation form: (a) Rayleigh friction, (b) radiative relaxation, and (c) hypodiffusion. Bold lines denote total energy; light lines denote energy in the azonal wavenumber sector, $m>n / 5$. The insets show the case $\varepsilon_{0}=0.1 \times 10^{-6}$ vs time from 0 to $10^{5}$ days; note the $y$ axis now extends to $10^{4}$.

linear growth out to $t=10^{5}$, corresponding to the increasing equatorial jet seen in Fig. 4a. Finally, for the case of hypodiffusion we find that equilibrium is eventually reached in all cases, but that the time required for this depends on the energy input rate. For weak energy input the total energy is still growing with time at $t=$ 10000 , whereas for strong energy input equilibration occurs earlier. The reason for this dependence is the scale-selective nature of the dissipation: for weak energy input it takes longer for the motion to organize into larger-scale flow on which the dissipation can act. Note, however, that even in the weak input cases, equilibration still occurs (see inset in Fig. 4).

In conclusion, Rayleigh friction is the most convenient form of dissipation, in that the total energy is easily determined, but is more difficult to justify as a dissipation mechanism for the atmospheres of the giant planets. Radiative relaxation is the most physically motivated, but increases the time required to reach equilibrium. In the following we are interested in equilibrium states and how these depend on the main physical parameters of the system, for which radiative relaxation is less suitable. We therefore perform the main investigation using hypodiffusion, which has similarities to radiative relaxation in midlatitudes but allows a more rapid equilibration of the flow at low latitudes.

\section{Dependence on $\varepsilon_{0}$ and $L_{D}$}

With the aforementioned sensitivities in mind, we now proceed to consider how the basic properties of the equilibrated flow depend on the physical parameters of the system. In the following, we present results for the hypodiffusion case with $\delta_{l}=-1$ and $\nu_{l}=0.01$ in (15), and with $\delta$-correlated forcing, except for occasional, explicitly stated, comparisons with Rayleigh friction. Having fixed the form of the forcing and dissipation, the problem is now defined entirely in terms of the physical parameters $L_{D}$ and Ro, the latter being controlled through the energy input $\varepsilon_{0}$.

\section{a. Dependence on $\varepsilon_{0}$}

We first consider the dependence on the energy injection rate, $\varepsilon_{0}$, while holding all other quantities fixed. We focus on the case of large Rossby deformation radius, $L_{D}=10$, for which the system is similar to the $2 \mathrm{D}$ barotropic system. Figure 6 shows the zonal mean zonal velocity for the cases $\varepsilon_{0}=(100,10,1,0.1,0.01) \times 10^{-6}$. The most obvious feature is that the width of the bands decreases with decreasing $\varepsilon_{0}$. If we assume that the bandwidth should also scale as $n_{\mathrm{Rh}}$, defined as the scale at which anisotropy becomes important, then the jets present in Fig. 6 are consistent with (7): increasing $\varepsilon_{0}$ corresponds to reducing $2 a \Omega / U$, hence reducing $n_{\mathrm{Rh}}$. Note again, however, that, unlike the case when the large-scale dissipation has the form of a Rayleigh fric- 


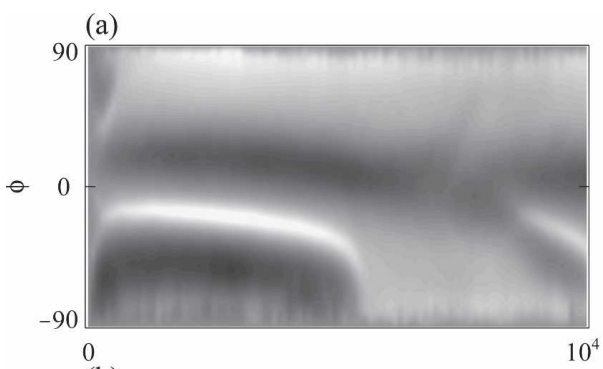

(b)
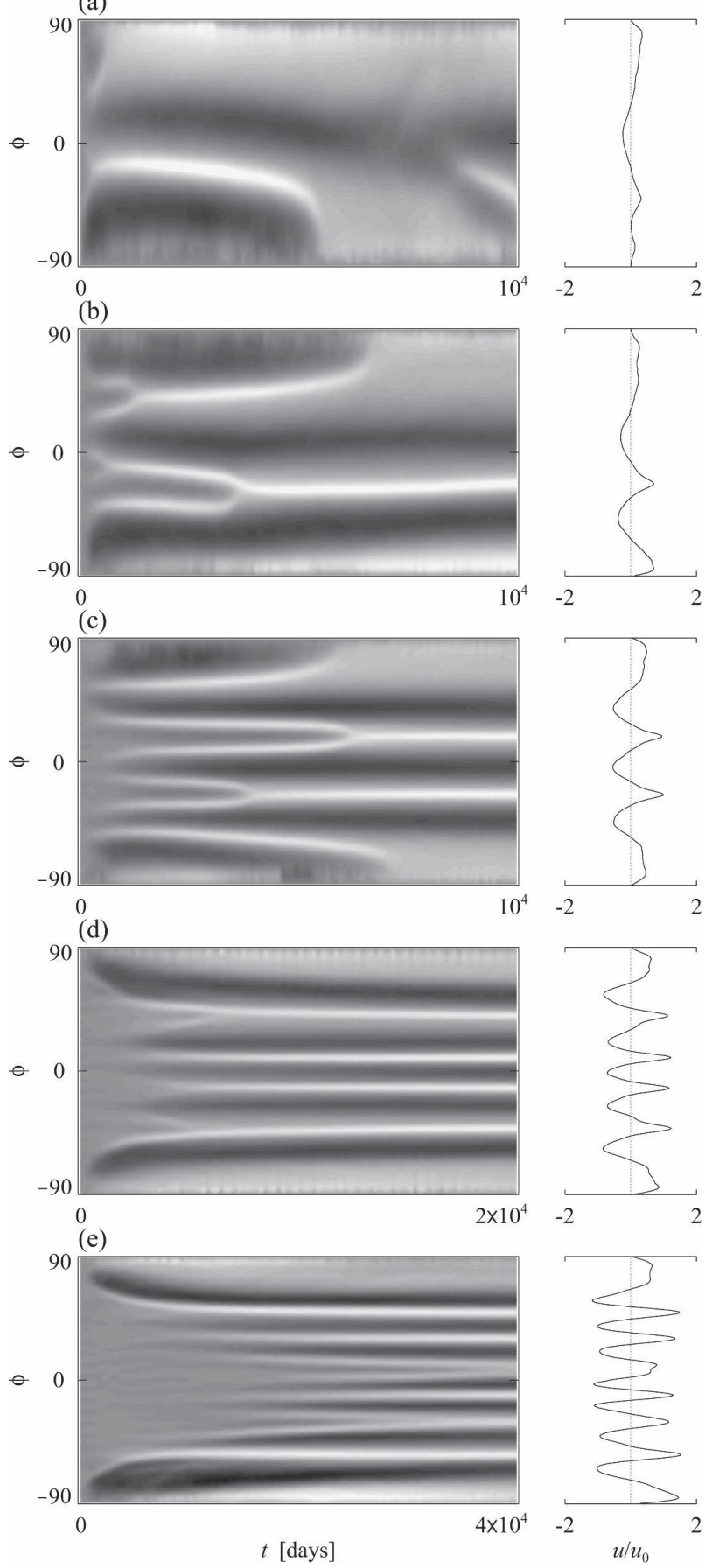

$-2$

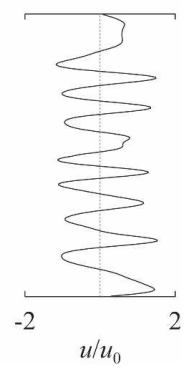

FIG. 6. Zonal velocity vs latitude and time (days) for the case $L_{D}=10$ and (a) $-(\mathrm{e}) \varepsilon_{0}=(100,10,1,0.1,0.01) \times 10^{-6}$. Gray scale is normalized according to the rms value, with light (dark) shade indicating eastward (westward) flow. Note the extended time scale in (d) and (e). In the profiles on the right, the zonal velocity $\bar{u}$ is normalized by $u_{0}=10^{2}\left(\varepsilon_{0}\right)^{1 / 2}$.
TABLE 1. Final Rossby number, predicted Rhines scale, and final energy centroid for the cases $L_{D}=10$ and $\varepsilon_{0}=(100,10,1$, $0.1,0.01) \times 10^{-6}$ (with integrations to $t=10000,10000,10000$, 20000 , and 40000 days, respectively).

\begin{tabular}{ccrc}
\hline$\varepsilon_{0} \times 10^{6}$ & Ro & $n_{\mathrm{Rh}}$ & $n_{0}$ \\
\hline 100 & 0.0179 & 3.7 & 4.7 \\
10 & 0.0080 & 5.6 & 6.6 \\
1 & 0.0037 & 8.2 & 8.8 \\
0.1 & 0.0016 & 12.4 & 13.3 \\
0.01 & 0.0007 & 19.3 & 17.0 \\
\hline
\end{tabular}

tion, we do not have an a priori estimate of $U$ based on $\varepsilon_{0}$. Note also that, since the equilibration takes longer at smaller $\varepsilon_{0}$, the last two calculations have been integrated out to $t=20000$ and $t=40000$, respectively (as opposed to $t=10000$ ). The longer equilibration time in these cases arises because the upscale energy cascade is stopped at higher wavenumbers where the hypodiffusion is weaker.

The simulated jet scale can be quantified by the energy centroid, defined as

$$
n_{0}=\frac{\int_{0}^{\infty} n E(n) d n}{\int_{0}^{\infty} E(n) d n},
$$

where $E(n)$ is the energy spectrum. This roughly corresponds to the peak in the energy spectrum at small $n$ and can be thought of as representing the largest scales at which energy accumulates. Table 1 lists the values of Ro $=U / 2 a \Omega$, the predicted Rhines scale $n_{\mathrm{Rh}}$, and the energy centroid, $n_{0}$, at the end of each simulation for different $\varepsilon_{0}$. For $n_{\mathrm{Rh}}$ we have used (3) as a local Rhines scale together with the approximation that $\beta=\beta_{\mathrm{rms}} \equiv$ $\Omega$. The scaling of two quantities $n_{0}$ and $n_{\mathrm{Rh}}$ for different values of $\varepsilon_{0}$ are in good agreement over a wide range of Ro. That is, from the velocity scale $U$, the Rhines scale $n_{\mathrm{Rh}}$ provides a good estimate for the equilibrated energy centroid $n_{0}$. Further, by fitting to these data points, we can estimate a power-law dependence between Ro and the energy input rate, $\operatorname{Ro} \sim \varepsilon_{0}^{\gamma}$, for which we obtain $\gamma \approx 0.36$. This yields $n_{0} \sim \varepsilon_{0}^{-0.18}$, which is in good agreement with (8), the predicted Rhines scale based on the upscale energy transfer.

Finally, we note that already in the zonal mean velocity an asymmetry can be discerned between eastward and westward jets, the former being in general somewhat stronger and narrower than the latter. This is most evident in intermediate cases (e.g., $\varepsilon_{0}=10,1$, $\left.0.1 \times 10^{-6}\right)$. This zonal structure arises naturally from the homogenization of $\mathrm{PV}$ into a staircase structure 
TABLE 2. The dependence of final values of $\alpha=\operatorname{Ro} / L_{D}^{2}$ and energy centroid $n_{0}$ on the values of $\varepsilon_{0}, L_{D}$ constituting the main parameter sweep.

\begin{tabular}{cccccc}
\hline \hline & \multicolumn{5}{c}{$\varepsilon_{0} \times 10^{6}$} \\
\cline { 2 - 5 }$L_{D}$ & 0.01 & 0.1 & 1 & 10 & 100 \\
\hline 10 & $\alpha=0.00001$ & $\alpha=0.00002$ & $\alpha=0.00004$ & $\alpha=0.00008$ & $\alpha=0.00002$ \\
& $n_{0}=17.0$ & $n_{0}=13.3$ & $n_{0}=8.8$ & $n_{0}=6.6$ & $n_{0}=4.7$ \\
1 & $\alpha=0.0007$ & $\alpha=0.002$ & $\alpha=0.004$ & $\alpha=0.009$ & $\alpha=0.02$ \\
& $n_{0}=17.6$ & $n_{0}=14.7$ & $n_{0}=8.7$ & $n_{0}=7.0$ & $n_{0}=4.6$ \\
0.1 & $\alpha=0.07$ & $\alpha=0.2$ & $\alpha=0.4$ & $\alpha=0.7$ & $\alpha=2.0$ \\
& $n_{0}=18.8$ & $n_{0}=16.0$ & $n_{0}=10.8$ & $n_{0}=5.4$ & \\
0.03 & $\alpha=0.6$ & $\alpha=1.5$ & $\alpha=3.3$ & & \\
& $n_{0}=19.7$ & $n_{0}=17.5$ & $n_{0}=14.1$ & \\
0.01 & $\alpha=2.7$ & $\alpha=6.1$ & & & \\
& $n_{0}=21.7$ & $n_{0}=13.5$ & & & \\
\end{tabular}

(Dritschel and McIntyre 2007; Dunkerton and Scott 2007; McIntyre 1982), the sharp eastward jets corresponding to jumps in the PV, and the broad zones of westward flow corresponding to zones of homogenized $\mathrm{PV}$. This structure will be considered further in section 7 below.

\section{b. Dependence on $L_{D}$}

Next, we consider how the nature of the equilibrated flow changes as the deformation radius is reduced from $L_{D}=10$. Table 2 illustrates the range of the parameters $\varepsilon_{0}$ and $L_{D}$ used. For each case we show the quantities $\alpha=\operatorname{Ro} / L_{D}^{2}$ and the energy centroid $n_{0}$. We note that we are unable to reduce $L_{D}$ arbitrarily, since in shallowwater deviations of the free surface become large with increasing Froude number, $\mathrm{Fr}=a \mathrm{Ro} / L_{D}$. Because of this, we have been unable to compute solutions for $L_{D}$ below values of about 0.01 , particularly for cases with larger Ro.

From (7) we do not expect the formation of anisotropic motion for $\alpha \geq 2$. The situation is complicated, however, by the fact that, although the upscale cascade of total (kinetic and potential) energy continues to ever-larger scales, the upscale cascade of kinetic energy will not proceed beyond $L_{D}$ (Polvani et al. 1994). The forcing here is at a length scale $L_{f}=a / n_{f} \approx 0.024$, which is greater than our smallest $L_{D}$, and so in these cases we do not expect any upscale transfer of kinetic energy. In fact, we do not find that the equilibrated zonal jet scale depends significantly on $L_{D}$ (see Table 2). There is a clear dependence of $n_{0}$ on Ro similar to that in the previous section, at each $L_{D}$ considered, but for a given Ro, $n_{0}$ is approximately uniform in $L_{D}$.

The influence of $L_{D}$ only becomes apparent when we consider the latitudinal structure of the zonal velocity field, shown in Fig. 7 for a particular $\varepsilon_{0}$. As $L_{D}$ becomes small we obtain a clear illustration of the regime discussed recently by Theiss (2004), where the jets in low latitudes persist but those in high latitudes disappear (see Fig. 7). This latitudinal structure is attributed to the latitudinal variation of the local $L_{D}$ on the sphere. For very small $L_{D}$, (7) suggests isotropy, since $\alpha=$ $\mathrm{Ro} / L_{D}^{2}$ is large and $n_{\mathrm{Rh}}$ is not defined. According to Theiss (2004) we may therefore expect a difference between equatorial regions, where the local $L_{D}$, given by the equatorial deformation radius $L_{\mathrm{eq}}=\left(a L_{D}\right)^{1 / 2}$ is larger, and midlatitude regions, where $L_{D}$ is smaller. We may also expect that for larger global $L_{D}$, jets will occur over a wider latitudinal range. This behavior is demonstrated clearly in the progression shown in Fig. 7. As predicted, the banded structure in the zonal mean zonal velocity is confined to lower latitudes for smaller $L_{D}$. Note again that the actual width of the bands themselves varies only weakly with $L_{D}$.

The way in which the jets initially form at early times also depends on $L_{D}$. At large $L_{D}$ jets form first at high latitudes. As $L_{D}$ decreases and the jets become confined to low latitudes, the initial jet formation at high latitudes is also retarded. Again, this is most apparent at small $\varepsilon_{0}$ (cf. Fig. 7c and Fig. 7d).

Finally, we point out that the low-latitude confinement of the jets is a robust feature arising from the latitudinal variation of $L_{D}$, and that it is not an artifact of the particular form of large-scale dissipation. In Fig. 8 we show the final zonal mean velocity profile for simulations the same as those used to obtain Figs. 7c-e but with the hypodiffusion replaced by Rayleigh friction. All other physical and numerical parameters are identical. Again we find the same low-latitude confinement as before, with good agreement between both of the jet scales, and the width of the confinement. The former is explained by the fact that the Rossby numbers 
(a)

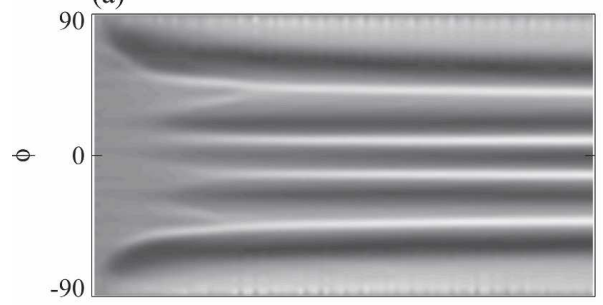

(b)

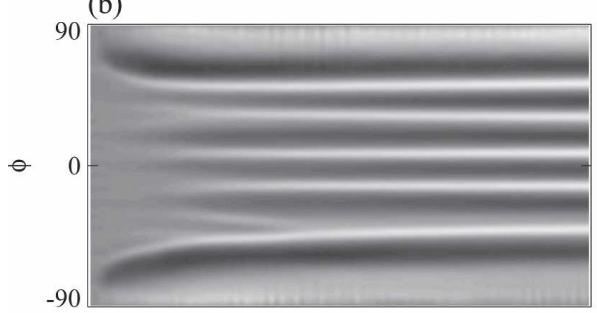

(c)

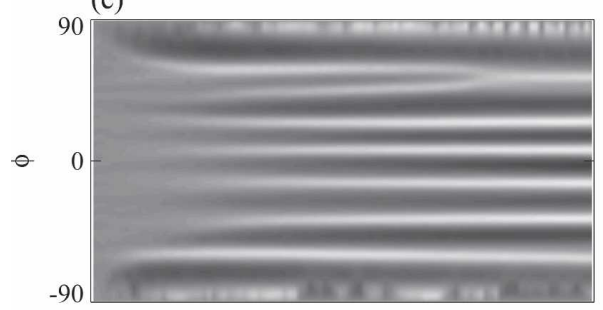

(d)

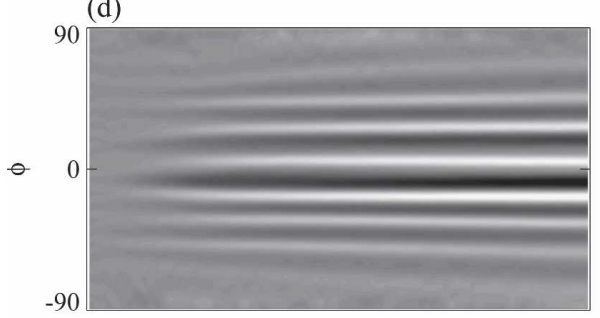

(e)

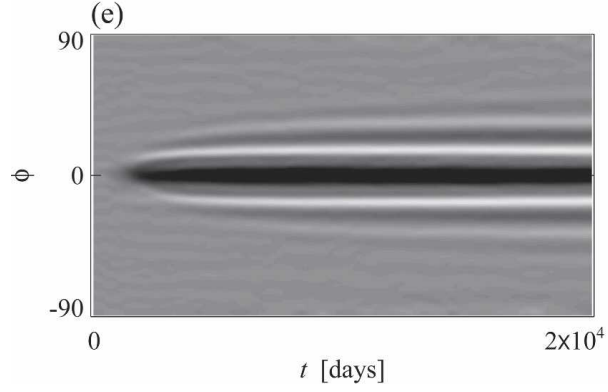

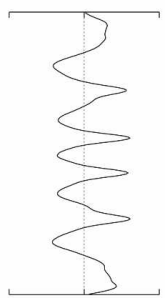
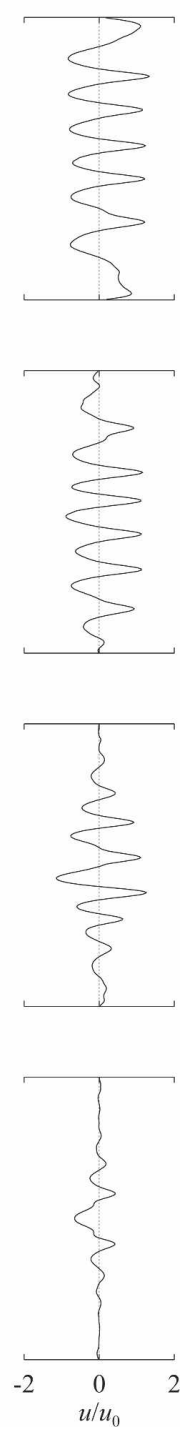

Fig. 7. Zonal velocity vs latitude and time (days) for the case $\varepsilon_{0}=0.1 \times 10^{-6}$ and (a)-(e) $L_{D}=10,1,0.1,0.03,0.01$. Gray scale is normalized according to the rms value, with light (dark) shade indicating eastward (westward) flow. Note that the time and velocity scales for (a)-(d) are listed in (e). In the profiles on the right, $\bar{u}$ is normalized by $u_{0}=10^{2}\left(\varepsilon_{0}\right)^{1 / 2}$. (a)

(b)
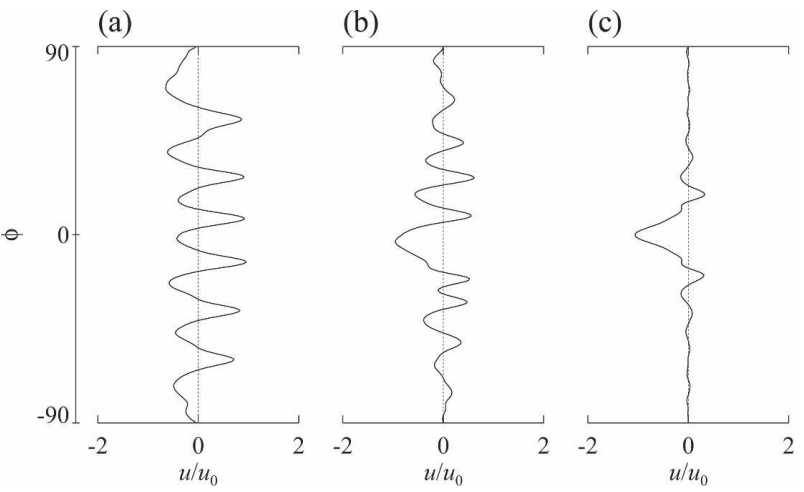

FIG. 8. Same as the velocity profiles in Figs. 7c-e, but with hypodiffusion replaced by Rayleigh friction (with friction coefficient $\nu_{l}=10^{-4}$ ): $L_{D}=$ (a) 0.1 , (b) 0.03 , and (c) 0.01 .

are similar between the two cases (to within about $25 \%$ ) and the latter is consistent with our hypothesis that the confinement is determined by $L_{D}$. Note that we have also performed a similar series of calculations in an equivalent barotropic version of the model in which the latitudinal variation of $L_{D}$ is absent. In that case no confinement was found: as $L_{D}$ is decreased there is a transition from a jet-dominated regime to a regime in which jets are completely absent.

\section{Turbulent properties}

We next consider some details of the turbulent structure of the simulations described above. A commonly used diagnostic for this is the kinetic energy spectrum. Here, however, the energy input wavenumber $n_{f}=42$, while for multiple jet regimes the energy centroid is around $n_{0}=10$ or larger, which means the range of the inverse energy cascade is very short. In cases where $n_{0}$ is smaller (see Table 2) an approximate power-law dependence of $E(n) \sim n^{-3.2}$ is obtained in the range $\left[n_{0}\right.$, $\left.n_{f}\right]$, but for larger $n_{0}$ no clear scaling was apparent, even using ensemble calculations. This is the case, regardless of whether hypodiffusion or Rayleigh friction is used as large-scale dissipation. The only robust feature that appears is that the energy contained in the azonal wavenumbers shows a reasonable $n^{-5 / 3}$ dependence in most cases. In general, however, power spectra for the multiple jet cases of most interest are not revealing. We therefore focus instead on the dynamically significant quantity of the potential vorticity, and consider its distribution in physical space.

\section{a. Potential vorticity staircases}

Figure 9 shows the relative and potential vorticity for the Northern Hemisphere for cases $\varepsilon_{0}=0.1 \times 10^{-6}$ and 
(a)

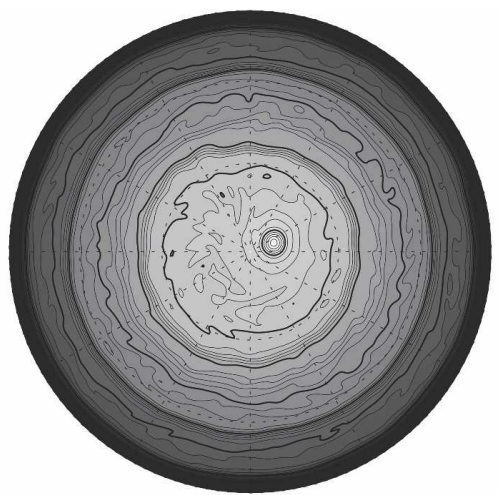

(d)

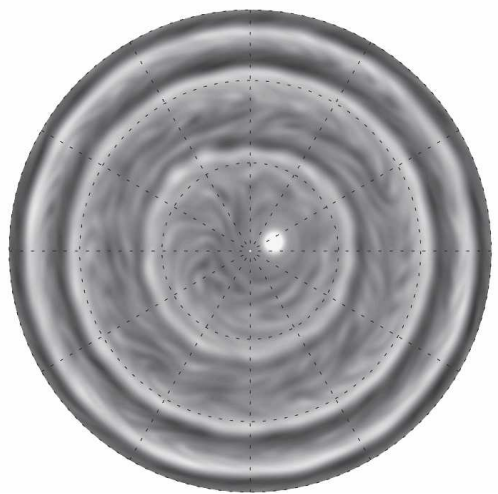

(b)

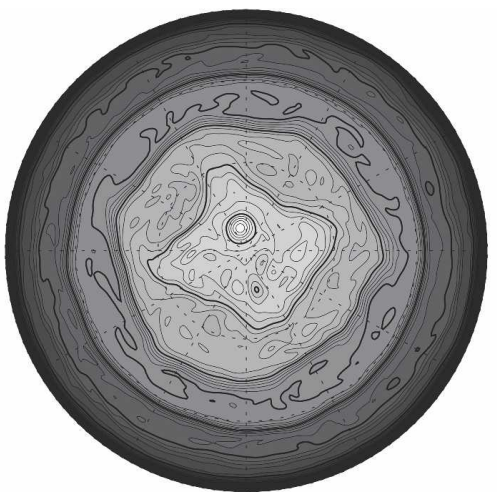

(e)

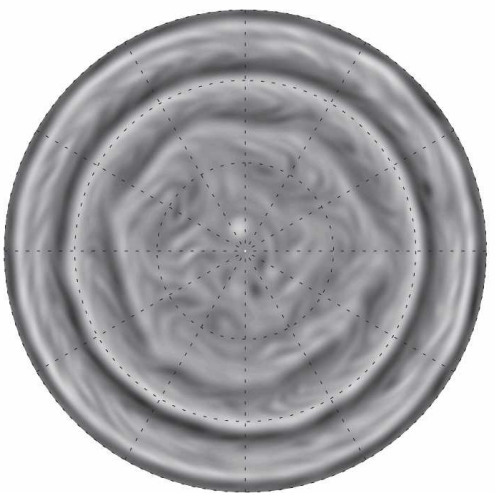

(c)

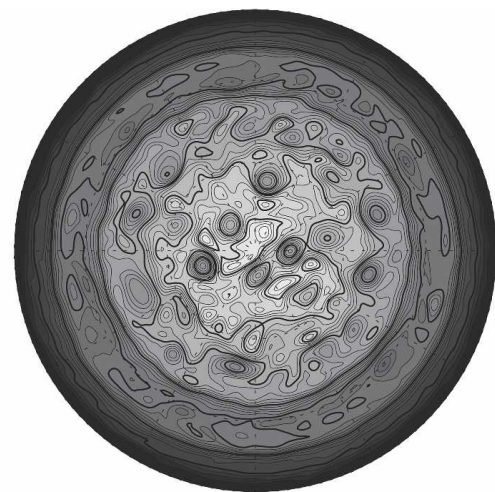

$(f)$

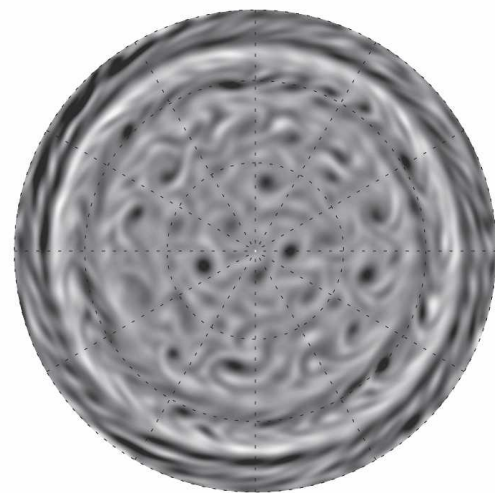

Fig. 9. (a)-(c) Potential vorticity and (d)-(f) vorticity fields at $t=20000$ days for the case $\varepsilon_{0}=0.1 \times 10^{-6}$ and $L_{D}=$ (a),(d) 0.1 , (b),(e) 0.03 , and (c),(f) 0.01 . Polar Lambert equal area projection. Light shading correspond to positive vorticity and high PV. In (a)-(c) the contour interval is $0.03 \times 4 \pi$ and the highest bold contour is $1.05 \times 4 \pi$ (nondimensional units).

$L_{D}=0.1,0.03$, and 0.01 (corresponding to Figs. 7c-e). Zonal jets are identified with bands of vorticity of alternating sign. In the PV field, the zonal jets appear as sharp jumps separating regions of almost zero PV gradient, where the corresponding zonal velocity is negative. The zonal mean PV profile (see Fig. 10) thus resembles a "staircase," and this structure is a well-known signature of PV mixing by the small-scale turbulent eddy field (Dritschel and McIntyre 2007; Dunkerton and Scott 2007; McIntyre 1982). As a result of this PV structure, the velocity profile exhibits an east-west asymmetry with strong, narrow eastward jets at the PV jumps separated by weak, broad westward jets between the jumps (see, e.g., Fig. 6c). We note that in all the simulations discussed in the preceding sections the presence of zonal jets is robustly accompanied by a staircase structure in the PV. The PV staircase is equivalent to a sawtooth structure in the relative vorticity, comprising broad regions of constant vorticity gradient separated by sharp jumps (e.g., Danilov and Gurarie 2004, for $\beta$-plane turbulence).

Figure 9 also shows how the zonality of the jets weak- ens with decreasing $L_{D}$. For $L_{D}=0.1$, the jets are almost perfectly zonal at all latitudes. For $L_{D}=0.03$, jets at low latitudes are zonal whereas those at high latitudes are increasingly undular. Even poleward of $60^{\circ}$ however, the PV contours remain intact and encircle the polar cap, despite being highly distorted. For (a)

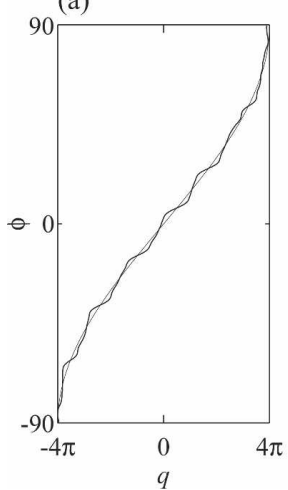

(b)

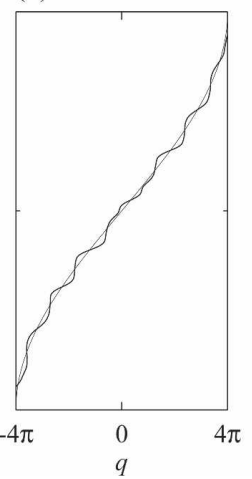

(c)

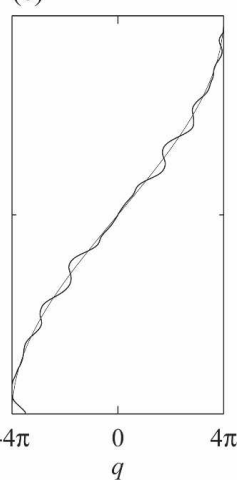

FIG. 10. Zonal mean potential vorticity vs latitude for the case $\varepsilon_{0}=1.0 \times 10^{-6}$ and (a)-(c) $L_{D}=0.1,0.03$, and 0.01 . 
$L_{D}=0.01$, these undular PV contours are finally broken at high latitudes, and blobs of low PV penetrate all the way into polar regions. PV is mixed across the polar cap and the flow is largely isotropic poleward of around $60^{\circ}$. The strongly zonal jets at large $L_{D}$ or low latitudes stand in contrast to the often meandering or spiraling jets arising in freely decaying turbulence (e.g., Cho and Polvani 1996a). Note the presence of a single strong cyclone at the pole in the cases $L_{D}=0.1$ and $L_{D}=0.03$ (the prominent white dot in Figs. 9a,d).

In Fig. 10c, it can be seen that the PV gradient in midlatitudes is negative between jumps. In a barotropic flow such a PV structure would of course be linearly unstable. However, we are aware of no rigorous stability criteria for shallow-water flow on the sphere, so nothing can be said about the stability of the zonal jets in this case. From the point of view of PV mixing it is nevertheless surprising that persistent negative gradients are maintained. The negative gradients can be seen to arise from coherent vortices situated near $20^{\circ}$ and $50^{\circ}$ (Fig. 9c).

Finally, Fig. 11 shows the vorticity for a large Ro case for which there are no jets in the Northern Hemisphere (cf. Fig. 6b). The PV in this case is dominated by a single strong cyclone at the pole. At midlatitudes, the PV decreases relatively smoothly toward the equator. The PV contours are also highly undular in this case, reflecting the stronger eddy activity of the flow. The stronger eddy mixing attempts to mix PV across the whole hemisphere, preventing the formation of discrete PV jumps and multiple jets. Yet it is unable to mix completely and the background planetary vorticity gradient remains dominant.

\section{b. Cyclone-anticyclone asymmetry}

The shallow-water system is distinguished from the barotropic or equivalent barotropic systems by the presence of an asymmetry between cyclonic and anticyclonic vorticity. As demonstrated by Polvani et al. (1994) and Stegner and Dritschel (2000), anticyclones tend to be more stable than cyclones and therefore the evolution of turbulent flow tends to favor the former. The criterion for the development of such asymmetry was determined empirically in numerical simulations of freely decaying shallow-water turbulence on the sphere by Cho and Polvani (1996a) and verified by Iacono et al. (1999a) as approximately $\mathrm{Ro} / L_{D}^{2} \geq 0.13$. Both studies considered the skewness of the cyclonicity defined as $\zeta \operatorname{sgn}(\phi)$. The $\operatorname{sgn}(\phi)$ is necessary on the sphere since positive vorticity is cyclonic in one hemisphere and negative in the other. The vorticity fields shown in Fig. 9 and Fig. 11 also indicate a strong degree of asymmetry (a)

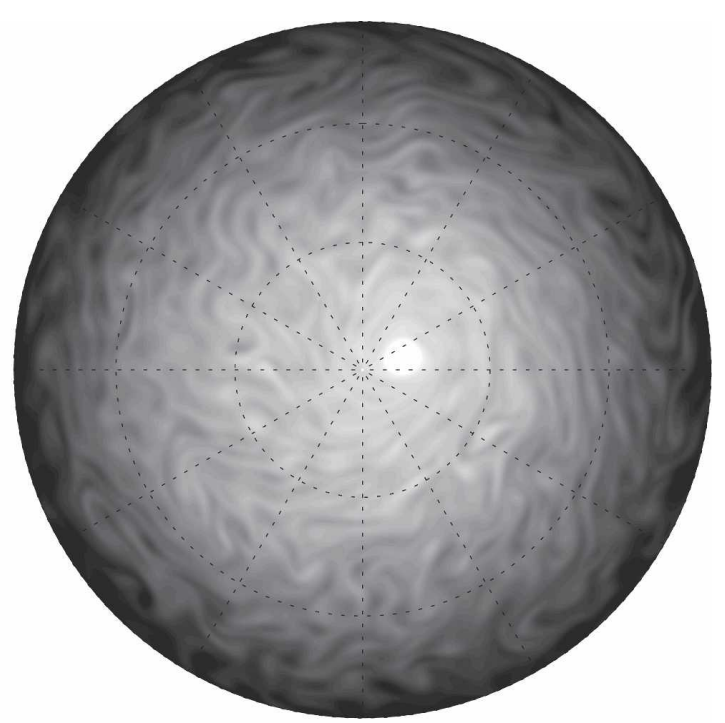

(b)

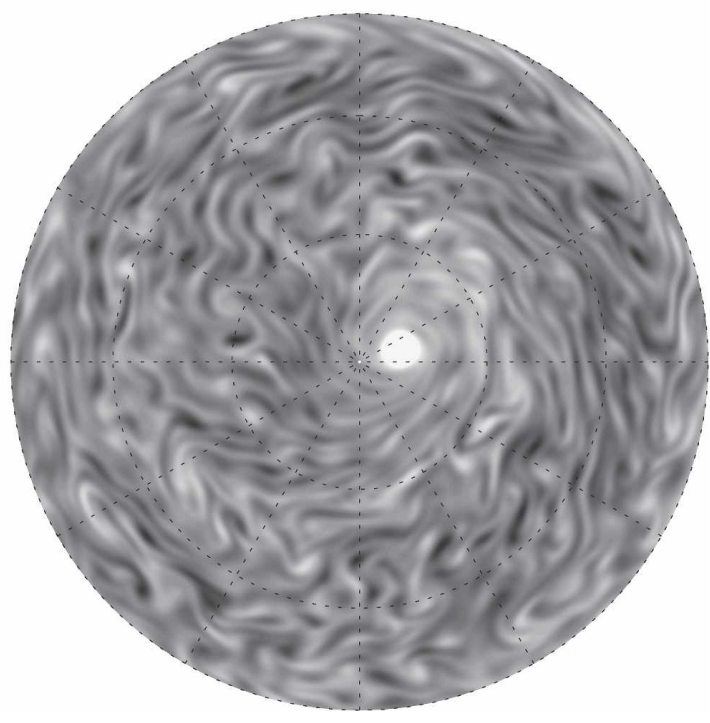

FIG. 11. (a) Potential vorticity and (b) vorticity at $t=10000$ days for the case $\varepsilon_{0}=10 \times 10^{-6}$ and $L_{D}=10$.

between cyclonic and anticyclonic motions: for example, the single intense cyclone in polar regions. In Fig. $9 \mathrm{c}$ intense coherent vortices in mid to high-latitude regions are predominantly anticyclonic, similar to $f$ plane shallow-water turbulence.

Figure 12 shows the skewness $\mathrm{Sk}$ as a function of time for all cases listed in Table 2, where

$$
\operatorname{Sk}(C)=\frac{\left\langle(C-\langle C\rangle)^{3}\right\rangle}{\left\langle(C-\langle C\rangle)^{2}\right\rangle^{3 / 2}},
$$

and where $C=\zeta \operatorname{sgn}(\phi)$ is the cyclonicity. In general, for large $L_{D}, \operatorname{Sk}(C)$ is always positive, that is, cyclones are on average more intense than anticyclones. This is 


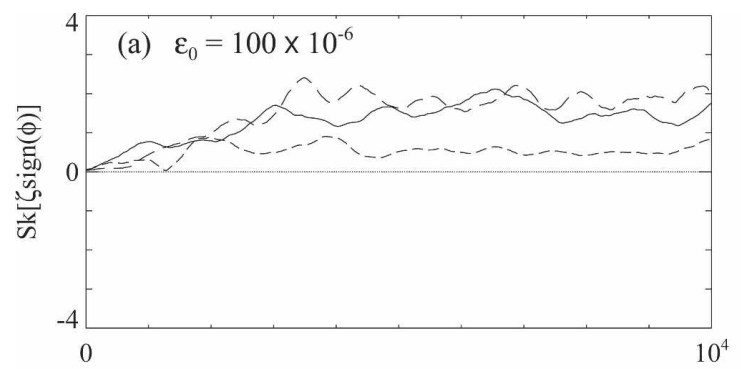

(b) $\varepsilon_{0}=10 \times 10^{-6}$
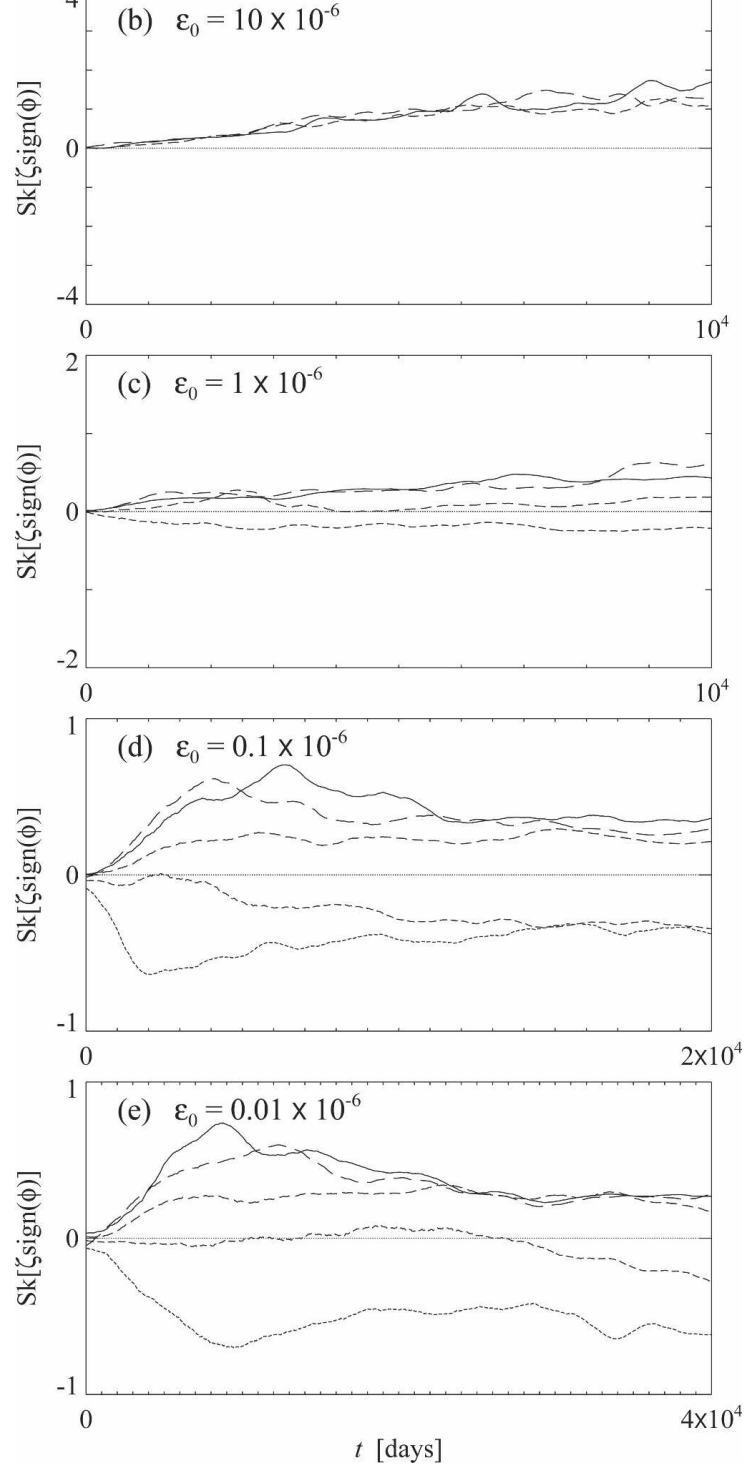

FIG. 12. Skewness of the cyclonicity field, $\zeta \operatorname{sgn}(\phi)$, vs time (days) for the cases (a)-(e) $\varepsilon_{0}=(100,10,1,0.1$, and 0.01$) \times 10^{-6}$ and $L_{D}=10,1,0.1,0.03$, and 0.01 (solid and decreasing dash lengths). The case $L_{D}=0.01$ is absent in (a)-(c) and the case $L_{D}=0.03$ is absent in (a), (b). in contrast to the freely decaying case where for large $L_{D}$ the skewness tends to be close to zero and there is no asymmetry. In the forced-dissipative case, the continual injection of enstrophy appears to accumulate in strong cyclonic polar vortices and midlatitude bands, to a greater extent than is possible in the freely decaying case. As $L_{D}$ decreases there is a transition to negative skewness, similar to that observed in the freely decaying case. Further, at smaller $\varepsilon_{0}$ the transition is abrupt, consistent with freely decaying studies (Cho and Polvani 1996a; Iacono et al. 1999a). The transition coincides with the disappearance of the cyclonic polar vortex and midlatitude bands (cf. Fig. 9).

In summary, the transition between symmetric and asymmetric evolution is similar to the freely decaying case with the difference that at large $L_{D}$ the flow is predominantly cyclonic, rather than purely symmetric. The transition to predominantly anticyclonic flow only occurs at small Ro, at least within the parameter range consistent with the shallow-water approximation, consistent with the freely decaying case (Cho and Polvani 1996a).

\section{Relevance to planetary atmospheres}

Having explored the full parameter space in a general context, we return to the circulation of the giant planets. We present results from three simulations, using parameters similar to those estimated for the giant planets (values taken from Cho and Polvani 1996b): Jupiter $\left(\mathrm{Ro}=0.002, L_{D}=0.025\right)$; Saturn $(\mathrm{Ro}=0.013$, $\left.L_{D}=0.025\right)$; and Uranus/Neptune (Ro $=0.06, L_{D}=$ 0.1 ). The zonal mean velocity profiles are shown in Fig. 13 and the PV and vorticity are shown in Fig. 14. The magnitudes of the zonal mean velocity profiles are comparable to those of the planets. Further, the magnitude of the equatorial flow is larger than that of midlatitude flow, in common with all four planets. However, the simulated equatorial flow is always retrograde, as in Cho and Polvani (1996b), whereas it is prograde on Jupiter and Saturn. Therefore, while the forced calculations are in agreement with some aspects of the planetary circulations, they do not appear to improve on the main shortcomings of the freely decaying model.

The vorticity and potential vorticity plots illustrate strong mixing over the equatorial regions in all cases. In midlatitudes the flow is more undular than on the planets, for example at $30^{\circ} \mathrm{S}$ in the Jupiter case. In the Saturn case, intense anticyclones are visible in midlatitudes, which dominate any jet structure poleward of the prograde jets at $\pm 20^{\circ}$. Intense anticyclones are a feature of the atmospheres of both Jupiter and Saturn. In 
(a)

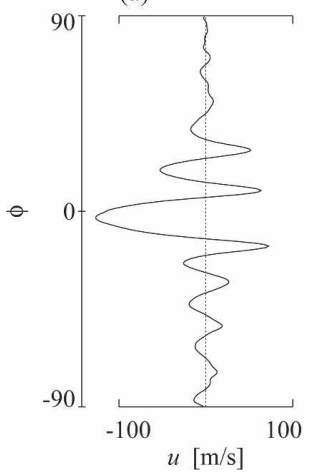

(b)

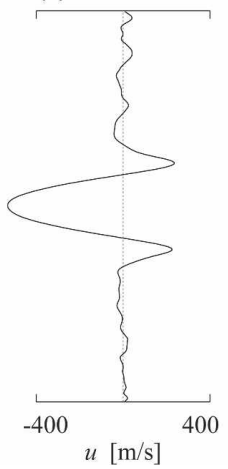

(c)

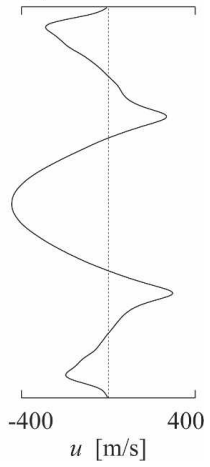

FIG. 13. Mean zonal velocity vs latitude for three simulations with planetary parameters: (a) Jupiter $\left(\mathrm{Ro}=0.002, L_{D}=0.025\right)$, (b) Saturn $\left(\mathrm{Ro}=0.013, L_{D}=0.025\right)$, and (c) Uranus/Neptune $\left(\mathrm{Ro}=0.06, L_{D}=0.1\right)$.

the Uranus/Neptune case, the potential vorticity is well mixed all the way out to $\pm 45^{\circ}$, where there are symmetric prograde jets, which are highly zonal.

In section 5 , equatorial confinement was found to occur for $L_{D}$ below around 0.03 . Consistent with this, the jets in the Jupiter and Saturn simulations are also restricted in latitude and the equatorial flow is considerably stronger than that at higher latitudes. However, we have found that at all $L_{D}$ for which there is equatorial confinement, the equatorial flow is retrograde while prograde equatorial jets only occur at larger $L_{D}$ [in the barotropic system, $L_{D} \rightarrow \infty$, prograde and retrograde jets appear with equal likelihood (Dunkerton and Scott 2007)]. Further, as $L_{D}$ decreases the zonal jets at high latitudes become increasingly undular. In contrast, jets on the giant planets appear remarkably zonal even at high latitudes. Thus it does not appear that low-latitude confinement due to small $L_{D}$ is the reason for the stronger equatorial flow of the giant planets.

From Figs. 13 and 14 it appears that adding a spatially uniform, homogeneous forcing does not change significantly the key features that emerge in the freely decaying calculations of Cho and Polvani (1996b), notably the uniformly retrograde jets at the equator. In another article in this special issue Showman (2007) presents shallow-water calculations representative of the Jovian atmosphere, using a model of thermal convection as (a)
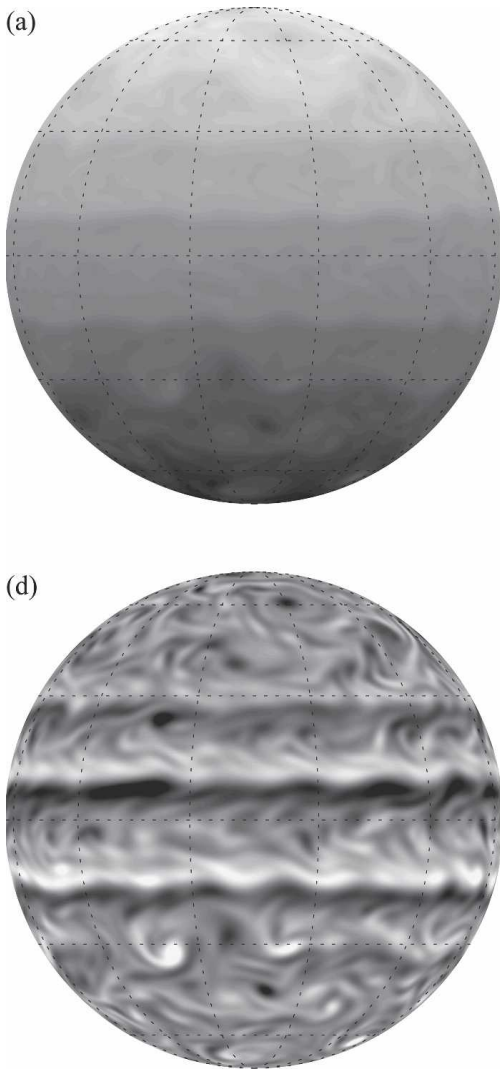

(b)

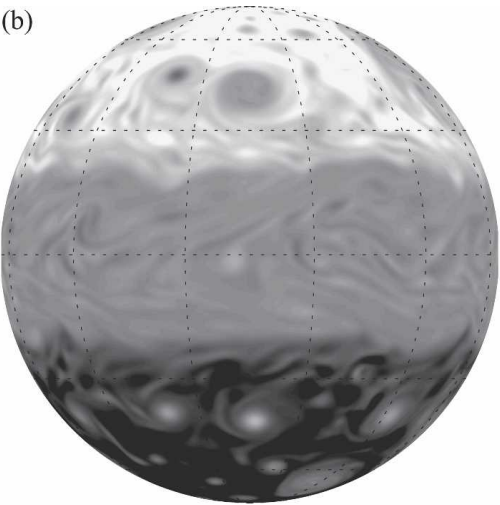

(e)

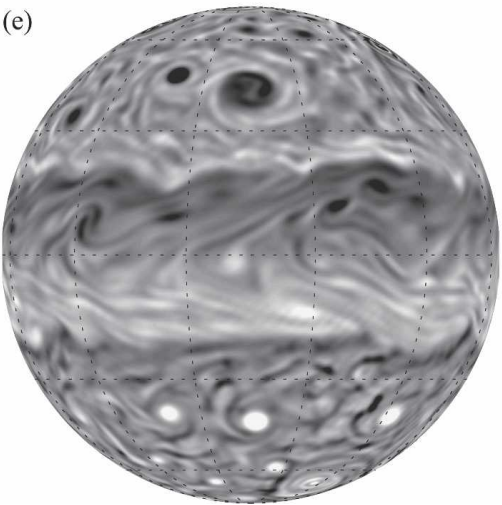

(c)

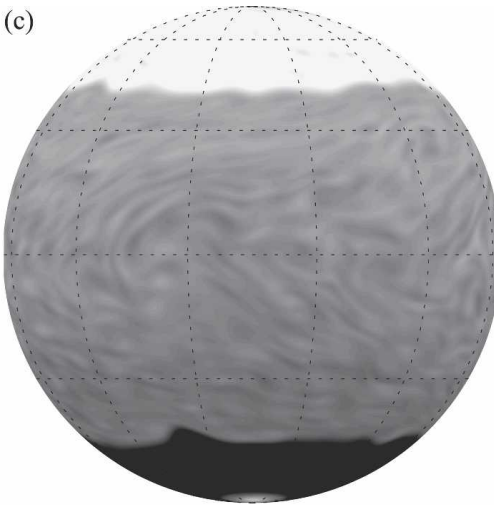

(f)

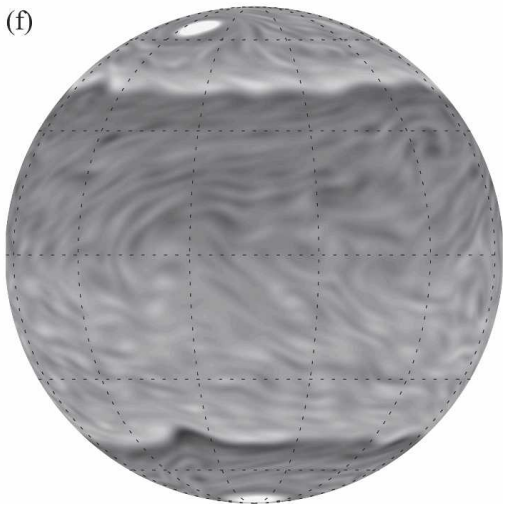

FIG. 14. (a)-(c) Potential vorticity and (d)-(f) vorticity for the three simulations with planetary parameters: (left) Jupiter, (middle) Saturn, and (right) Uranus/Neptune. Shading is same as in Fig. 9. 
small-scale forcing and radiative relaxation as largescale dissipation. As in the present case, Showman finds retrograde equatorial jets and jet-dominated flow restricted to low latitudes for $L_{D}$ comparable to the Jovian value. The two sets of calculations appear to be fully consistent with each other, and suggest that some other physical processes, beyond those contained in the shallow-water system, may be needed to capture the main features of the giant planets.

In section 4, it was seen that when the large-scale dissipation takes the form of a radiative relaxation, zonal momentum can accumulate in the Tropics to a greater extent than in the extratropics, resulting in a stronger equatorial jet. At large $L_{D}$ the jet can also be prograde (Fig. 4a). One question, therefore, is whether the jet structure may be governed by a larger $L_{D}$ than that relevant to the coherent vortices from which estimates of the planetary values of $L_{D}$ have been made. This would be the case, for example, if the jets were much deeper than the vortices, as suggested by the Galileo probe (Atkinson et al. 1998), since the deformation radius relevant to shallower structures is that based on higher modes of the vertical structure equation. In fact, preliminary calculations suggest that radiative relaxation can also give rise to prograde equatorial jets even at smaller $L_{D}$, and a more complete investigation of this form of dissipation is currently under way.

\section{Summary and conclusions}

Across a wide range of physical and numerical parameters persistent zonal jets appear as a robust feature of the forced-dissipative shallow-water system in spherical geometry. To a good approximation the energy centroid (a measure of the jet scale) in the barotropic limit satisfies the power-law dependence $n_{0} \sim$ $\varepsilon^{-1 / 5}$ suggested by Maltrud and Vallis (1991). The variation of the jet scale with deformation radius is in general much weaker. In all cases the jet structure is accompanied by a latitudinal staircase profile in the PV indicating that the dynamics arises as a result of eddy mixing. While both prograde and retrograde equatorial jets are found to emerge at large deformation radius, only retrograde equatorial jets emerge at small deformation radius.

Our simulations provide a clear demonstration in full spherical geometry of the new regime proposed recently by Theiss (2004), where the zonal jet structure is confined to low latitudes at small deformation radius. Because of the dependence on $L_{D}$, this regime has not been observed in the spherical barotropic system. Independent work by the authors using an ad hoc equiva- lent barotropic model in spherical geometry (where $L_{D}$ is independent of latitude) also showed no low-latitude confinement, supporting the claim that the regime arises because of the latitudinal variation of $L_{D}$. The arguments in Theiss (2004) provide a simple physical motivation of the equatorial confinement, whereby the Rhines effect is suppressed at high latitudes because of the small local $L_{D}$, but is not suppressed at low latitudes because the local, equatorial deformation radius is larger. A suggestion of the same low-latitude confinement is apparent in earlier simulations of freely decaying shallow-water turbulence on the sphere (Cho and Polvani 1996a; Iacono et al. 1999b), although it was not reported as such. Here, the confinement was robustly obtained for both Rayleigh friction and hypodiffusion as large-scale dissipation, as well as for different forcing forms and numerical resolution.

Cyclone-anticyclone asymmetry was also observed in the forced-dissipative shallow-water system. Unlike the freely decaying case, at large $L_{D}$ the skewness of the cyclonicity is positive at all Rossby numbers considered indicating more intense cyclonic eddies. This result is consistent with the $\beta$ drift of vortices, where cyclonic vortices tend to drift poleward and anticyclonic vortices tend to drift equatorward. As $L_{D}$ decreases, however, shallow-water dynamics departs from equivalent barotropic dynamics and favors anticyclonic vorticity, consistent with the freely decaying case (Cho and Polvani 1996a). More intense anticyclones were also observed in the small $L_{D}$ cases presented here, particularly in polar regions.

Although the zonal jets obtained here are in general quasi-steady, the degree of steadiness depends on the balance between forcing and dissipation. For a given total energy, it was shown that the steadiness of the jets decreases with increasing forcing and dissipation magnitude. On the other hand, the steadiness is independent of the form of the large-scale dissipation. A more physically relevant dissipation operator, representing radiative relaxation, was also considered. In this case, the evolution was characterized by the development of strong equatorial jets, a consequence of the weak constraint of radiative relaxation on angular momentum at low latitudes.

Returning to the application to the giant planets we conclude that the inclusion of homogeneous, isotropic forcing in the shallow-water system is still insufficient to explain all of the observed features of those atmospheres. The main advantage over the freely decaying case considered by Cho and Polvani may be simply that the jet structure is more clearly defined. At small $L_{D}$, the equatorial jet is still consistently retrograde, at least 
when hypodiffusion or Rayleigh friction is used as large-scale dissipation, and the high-latitude flow is more undular than is observed on Jupiter and Saturn. However, with regard to the forcing and dissipation, the present study is far from exhaustive and it may still turn out that very specific choices of these are needed to fully replicate the planetary features.

Acknowledgments. The authors wish to thank Tim Dunkerton, Michael McIntyre, and Jurgen Theiss for interesting discussions and comments on the manuscript, and an anonymous reviewer for helpful comments. Computations were carried out on supercomputing facilities at the National Center for Atmospheric Research. Financial support was provided by the National Science Foundation.

\section{REFERENCES}

Atkinson, D. H., J. B. Pollack, and A. Seiff, 1998: The Galileo probe Doppler wind experiment: Measurement of the deep zonal winds on Jupiter. J. Geophys. Res., 103, 22 911-22 928.

Borue, V., 1994: Inverse energy cascade in stationary twodimensional homogeneous turbulence. Phys. Rev. Lett., 72, $1475-1478$

Busse, F. H., 1976: A simple model of convection in the Jovian atmosphere. Icarus, 29, 255-260.

Cho, J. Y.-K., and L. M. Polvani, 1996a: The emergence of jets and vortices in freely-evolving shallow-water turbulence on a sphere. Phys. Fluids, 8, 1531-1552.

— , and — 1996b: The morphogenesis of bands and zonal winds in the atmospheres on the giant outer planets. Science, 273, 335-337.

_ M. de la Torre Jurez, A. P. Ingersoll, and D. G. Dritschel, 2001: A high-resolution, three-dimensional model of Jupiter's great red spot. J. Geophys. Res., 106, 5099-5105.

Danilov, S., and D. Gurarie, 2002: Rhines scale and spectra of the $\beta$-plane turbulence with bottom drag. Phys. Rev. E, 65, doi:10.1103/PhysRevE.65.067301.

—, and — 2004: Scaling, spectra and zonal jets in beta-plane turbulence. Phys. Fluids, 16, 2592-2603.

Dritschel, D. G., and M. E. McIntyre, 2007: Multiple jets as PV staircases: The Phillips effect and the resilience of eddytransport barriers. J. Atmos. Sci., in press.

Dunkerton, T. J., and R. K. Scott, 2007: A barotropic model of the angular momentum conserving potential vorticity staircase in spherical geometry. J. Atmos. Sci., in press.

Galperin, B., S. Sukoriansky, and H.-P. Huang, 2001: Universal $n^{-5}$ spectrum of zonal flows on giant planets. Phys. Fluids, 13, $1545-1548$

Garcia, R. R., 1987: On the mean meridional circulation of the middle atmosphere. J. Atmos. Sci., 44, 3599-3609.

Guillot, T., 1999: Interiors of giant planets inside and outside the solar system. Science, 286, 72-77.

Haynes, P. H., 1998: The latitudinal structure of the quasi-biennial oscillation. Quart. J. Roy. Meteor. Soc., 124, 2645-2670.

Heimpel, M., J. Aurnou, and J. Wicht, 2005: Simulation of equatorial and high-latitude jets on Jupiter in a deep convection model. Nature, 438, 193-196.
Huang, H.-P., and W. A. Robinson, 1998: Two-dimensional turbulence and persistent zonal jets in a global barotropic model. J. Atmos. Sci., 55, 611-632.

_, B. Galperin, and S. Sukoriansky, 2001: Anisotropic spectra in two-dimensional turbulence on the surface of a rotating sphere. Phys. Fluids, 13, 225-240.

Iacono, R., M. V. Struglia, and C. Ronchi, 1999a: Spontaneous formation of equatorial jets in freely decaying shallow water turbulence. Phys. Fluids, 11, 1272-1274.

,,,--- and S. Nicastro, 1999b: High-resolution simulations of freely decaying shallow-water turbulence on a rotating sphere. Nuovo Cimento, 22C, 813-821.

Ingersoll, A. P., and Coauthors, 2004: Dynamics of Jupiter's atmosphere. Jupiter: The Planet, Satellites and Magnetosphere, F. Bagenal, T. E. Dowling, and W. B. McKinnon, Eds., Cambridge Planetary Science Series, Vol. 1, Cambridge University Press, 105-128.

Maltrud, M. E., and G. K. Vallis, 1991: Energy spectra and coherent structures in forced two-dimensional and beta-plane turbulence. J. Fluid Mech., 228, 321-342.

McIntyre, M. E., 1982: How well do we understand the dynamics of stratospheric warmings? J. Meteor. Soc. Japan, 60, 37-65.

Nozawa, T., and S. Yoden, 1997: Formation of zonal band structure in forced two-dimensional turbulence on a rotating sphere. Phys. Fluids, 9, 2081-2093.

Okuno, A., and A. Masuda, 2003: Effect of horizontal divergence on the geostrophic turbulence on a beta-plane: Suppression of the Rhines effect. Phys. Fluids, 15, 56-65.

Polvani, L. M., J. C. McWilliams, M. A. Spall, and R. Ford, 1994: The coherent structures of shallow water turbulence: Deformation-radius effects, cyclone/anticyclone asymmetry and gravity-wave generation. Chaos, 4, 177-186, 427-430.

Rhines, P. B., 1975: Waves and turbulence on a beta-plane. $J$. Fluid Mech., 69, 417-443.

Rivier, L., R. Loft, and L. M. Polvani, 2002: An efficient spectral dynamical core for distributed memory computers. Mon. Wea. Rev., 130, 1384-1390.

Scott, R. K., 2007: Non-robustness of the two-dimensional turbulent inverse cascade. Phys. Rev. E, 75, doi:10.1103/PhysRevE. 75.046301

— , and P. E. Haynes, 1998: Internal interannual variability of the extratropical stratospheric circulation: The low-latitude flywheel. Quart. J. Roy. Meteor. Soc., 124, 2149-2173.

—, L. Rivier, R. Loft, and L. M. Polvani, 2004: BOB: Model description and user's guide. NCAR Tech. Note NCAR/TN456+IA, 30 pp.

Showman, A. P., 2007: Numerical simulations of forced shallowwater turbulence: Effects of moist convection on the largescale circulation of Jupiter and Saturn. J. Atmos. Sci., 64, 3132-3157.

Smith, K. S., 2004: A local model for planetary atmospheres forced by small-scale convection. J. Atmos. Sci., 61, 14201433.

Stanley, S., and J. Bloxham, 2004: Convective-region geometry as the cause of Uranus' and Neptune's unusual magnetic fields. Nature, 428, 151-153.

Stegner, A., and D. G. Dritschel, 2000: A numerical investigation of the stability of isolated shallow water vortices. J. Phys. Oceanogr., 30, 2562-2573.

Sukorianski, S., B. Galperin, and A. Chekhlov, 1999: Large scale drag representation in simulations of two-dimensional turbulence. Phys. Fluids, 11, 3043-3053.

,$- \ldots$, and N. Dikovskaya, 2002: Universal spectrum of two- 
dimensional turbulence on rotating sphere and some basic features of atmospheric circulations on giant planets. Phys. Rev. Lett., 89, doi:10.1103/PhysRevLett.89.124501.

- N. Dikovskaya, and B. Galperin, 2007: On the arrest of inverse energy cascade and the Rhines scale. J. Atmos. Sci., 64, 3312-3327.

Sun, Z.-P., G. Schubert, and G. A. Glatzmaier, 1993: Banded surface flow maintained by convection in a model of the rapidly rotating giant planets. Science, 260, 661-664.
Theiss, J., 2004: Equatorward energy cascade, critical latitude, and the predominance of cyclonic vortices in geostrophic turbulence. J. Phys. Oceanogr., 34, 1663-1678.

Williams, G. P., 1978: Planetary circulations: 1. Barotropic representation of Jovian and terrestrial turbulence. J. Atmos. Sci., 35, 1399-1424.

Yoden, S., and M. Yamada, 1993: A numerical experiment on two-dimensional decaying turbulence on a rotating sphere. $J$. Atmos. Sci., 50, 631-643. 